Fatima Hossain, MSc ${ }^{1}$, Nuno Ferreira, $\mathrm{PhD}^{2 *}$

\title{
Impact of Social Context on the Self-Concept of Gay and Lesbian Youth: A Systematic Review
}

\author{
'University of Edinburgh, 2. University of Nicosia \\ *email: ferreira.ndunic.ac.cy \\ DOI: 10.2478/gp-2019-0006 \\ Received: 24 January 2019; Accepted: 25 February 2019
}

\begin{abstract}
Objectives: Self-concept distortion has been extensively linked with decreasing mental health in gay and lesbian youth. Social context has been proposed to have a moderating effect on the development of a healthy self-concept. However, no good quality review has approached these concepts with regards to LGBT youth.

Methods: A systematic review was conducted on the relationship between social context and self-concept in gay and lesbian youth. Twenty studies were included in the review.

Results: Quality assessment of papers yielded moderate methodological strength. Findings implied that social context has considerable influence on self-concept development. Discrepancies in assessment methods, areas of social context examined, and one-dimensional nature of examining self-concept interferes with drawing explicit conclusions regarding the relationship between social context and self-concept.

Conclusion: Positivity of social context is not conclusively relatable to positive self-concept development, and similarly, a negative context is not predetermining of self-concept distortions. Building on resilience factors of gay and lesbian youth, working together with families, and advancing and utilizing available educational and community resources should mitigate the strength of overt and covert heterosexism hindering healthy self-concept development. Further longitudinal and cross-cultural research will be necessary to provide insight into the mechanisms of associations.
\end{abstract}

\section{Keywords}

gay, lesbian, youth, social context, self-esteem, self-concept, systematic review, adolescents, LGBT

\section{INTRODUCTION}

Gay and lesbian youth often face adverse reactions from their environment due to their "sexual minority status" (Garofalo et al., 1998). Sexual minority status is a concept that involves multiple elements: sexual attraction (the genders one is attracted to), behavior (the gender of one's sexual partners), and identity (label assigned to oneself) (Savin-Williams \& Diamond, 2000). Although there are a number of categories included within each element, the present work will focus on sexual attraction, and within that element, gay and lesbian youth.

Adolescence is a period of development, the course of which allows youth to explore their personal identities and begin to act according to gender roles acceptable by societal structures around them (Rotheram-Borus et al., 1995). During this time of development, gay and lesbian youth also have to come to terms with their sexual identities and examine what their sexual orientation means in relation to their personal identities (Rotheram-Borus et al., 1995). Forming and preserving a healthy self-concept while accepting one's belonging to such categories may present as problematic. Research has illustrated (e.g., Silverschanz et al., 2008; Woodford et al., 2012; Woodford et al., 2014) the significance of social context in providing a supportive background to overcoming hurdles and helping adjustments in the development of self-concept. Even though copious amount of research has explored these relationships, the existing body of literature is devoid of an overview of findings.

\section{Self-concept in gay and lesbian youth}

Although self-concept is commonly defined as a collection of beliefs about one's self (Campbell, 1990), clear definitions of self-concept will vary between authors. Bentall et al. (2001) 
cohesively reviewed the commonly used terminology around self-concept, identifying five areas that are commonly used to define self-concept:

self-esteem

self-consciousness

self-representations

self-schemas

self-perception

Development as an adolescent for gay and lesbian youth may feel even more difficult, as they also need to go through a process of constructing a healthy self-concept in a primarily heterosexual social environment (Rotheram-Borus et al., 1995). Each of the previously mentioned five areas is believed to be impacted in sexual minority youth by both internal and external contexts. Higgins' (1987) self-discrepancy theory helps us conceptualize the tensions of developing an LGBT (lesbian, gay, bisexual, and transgender) self-concept in a heteronormative context. Selfdiscrepancy theory proposes that individuals aim to achieve a position where their self-concept is in line with their socially learnt standards and ideals. According to self-discrepancy theory, if these conditions are not met, the person will be susceptible to feelings of fear, shame, rejection, and guilt, and high self-discrepancy would lead to low self-esteem and a negative self-concept.

Although ample studies concentrated on pinpointing how selfconcept develops and how it is influenced in youth, an emerging line of studies started to focus on identifying the directions and nature of relationship between both positive and negative beliefs about the self and others, and the social context, concentrating specifically on gay and lesbian youth (Vincke \& van Heeringen, 2002; Snapp et al., 2015; Wilkerson et al., 2017). For example, Vincke and van Heeringen (2002) examined a group of 197 gay and lesbian youth in a longitudinal study and found that when youth's environment, such as family, friends, and confidants, is aware of their sexual identity and is supportive and nurturing, gay and lesbian youth scored higher on self-concept measures. These findings are supported by Snapp et al. (2015) who studied 245 LGBT youth and showed that a positive and supportive environment (such as family, friends, and community) was associated with higher self-esteem in youth $(r=0.37)$ and also with a more positive attitude toward their sexual identities $(r=0.36)$. Findings of both studies were underpinned by recent findings by Wilkerson et al. (2017) who examined a sample of 108 sexual minority youth and found an association between a supportive social environment (such as family, friends, and LGBT youth organizations) and youth's self-esteem $(\beta=0.72$, $95 \%$ CI $[0.38,1.06])$.

\section{Social context}

It is suggested that an adverse and hostile social environment can negatively impact upon the well-being of sexual minority youth (Silverschanz et al., 2008; Woodford et al., 2012; Woodford et al., 2014).

Social context theory (Earle \& Earle, 1999) provides a framework for investigating the way self-concept in sexual minority youth may be affected by identifying and changing current social views of and attitudes toward gay and lesbian young people. Social context is a concept that provides an opportunity to assess perceptions of social support and acceptance, social behaviors and relationships, problems, decisions and dilemmas, and to establish models to resolve these through the incorporation of more inclusive attitudes and beliefs. The three key dimensions of social context theory are societal structures, social processes (perceptions, attitudes, values), and common patterns of social behaviors.

Out of the three dimensions, the most applicable in relation to sexual minority youth is the component of social processes (Garofalo et al., 1998). Gay and lesbian youth's sexual orientation does not conform to expected social norms and traditions and, as such, is commonly seen as danger to society, often producing discrimination, marginalization, and inequalities (Garofalo et al., 1998; Silverschanz et al., 2008; Woodford et al., 2012; Woodford et al., 2014).

\section{Social context and self-concept in gay and lesbian youth}

Social contexts in which youth live can have significant implications for their development and psychological adjustment (Garofalo et al, 1998). Generally perceiving others as hostile often leads to internalized homophobia, distorted selfperception, and decreased self-esteem, essentially hindering the development of a healthy self-concept (Rosario et al., 2011).

Literature suggests that adolescent development is further influenced by being gay or bisexual in an essentially heterosexual society (Sophie, 1986; Savin-Williams, 1990). The aforementioned studies found a positive relationship among social support, positive attitudes to homosexuality, and an increase in self-esteem. Generally, research has shown that social context is positively related to self-esteem (McNicholas, 2002; Detrie \& Lease, 2007). Sexual minority youth may perceive themselves as disconnected from the socially accepted majority heterosexual group, resulting in a lack of opportunity to take advantage of self-esteem built on membership in that group (Grossman, 1997). This view may suggest that homosexuality 
and deviance from expected gender-typed behaviors may lead to marginalization and may prevent the development of a positive and healthy self-concept (Rotheram-Borus, et al., 1995).

In a study of 106 sexual minority youth, Dahl and Galliher (2010) found moderate to large negative correlations between sexual orientation conflict and self-esteem, suggesting that individuals with higher orientation conflict may present lower self-esteem. In contrast, Bauermeister et al. (2010) examined 350 LGBT youth looking to establish an association between same-sex relationships and self-esteem. They found that being involved in same-sex relationships was positively associated with higher self-esteem in males; however, the same had no association with self-esteem in females.

Living in a supportive environment and being able to rely on positive social relationships can be a contributor to increased self-esteem, self-acceptance, and self-perception (Ensel \& Lin, 1991; Rosenfield \& Wenzel, 1997). This view is evidenced by studies that found a significant positive relationship between social support and self-esteem (D’Augelli \& Hershberger, 1993; Bagley \& Tremblay, 1997; van Heeringen \& Vincke, 2000).

In a longitudinal study of 197 gay and lesbian youth, it was found that social relationships can essentially be a source not only of tension and stress, but also of approval and support (Vincke \& van Heeringen, 2002). The authors also found that having someone to trust with their sexual orientation increased self-esteem in gay and lesbian youth. Similarly, examining a sample of 156 lesbian, gay, and bisexual youth, Rosario et al. (2005) reported a significant positive association between social desirability and self-esteem, as well as between social support from family and friends and self-esteem. Additionally, they showed a negative correlation between negative social relationships and self-esteem.

It is also suggested that concealing sexual orientation may delay the development of a positive self-concept in sexual minority youth (Bos et al., 2008), which can manifest in low selfesteem, rejection of one's sexuality, and increased internalized homophobia (conforming one's self-concept to be aligned with the stigmatizing reactions of society and developing a negative attitude toward the self as belonging to a stigmatized group) (Bauermeister et al., 2010). These findings were supported by Russel et al. (2014) who examined a sample of 245 LGBT youth and found that although being out at school (revealing one's sexual identity to others, such as family, friends, and peers) did not specifically impact on self-esteem, hiding sexual orientation did in fact show a significant negative association with self-esteem and with life satisfaction. These issues can negatively affect youth development and well-being, leading to not just personal issues, but also to a wider-spread overload and higher costs in the healthcare system. As in previous studies, Peplau and Fingerhut (2007) also reported that the presence of social support and social connectedness can lower the impact of distress caused by negative experiences.

Social context not only includes positive or negative attitudes of one's environment but also includes the social support received, as well as the perceived versus actual social attitudes. It is proposed (Sarason et al., 1987) that social support is multifaceted, that involves the perception of being of value to and loved by others, as well as the notion of being contented with the support received from one's environment. In providing these factors, especially important may be the youth's family and friends, school environment, and the wider community.

\section{School}

The influence of social context may also be observed in the school environment. These are settings that are often infused with heterosexism, homophobia, and generally overt gender rules, divergence from which is judged and frowned upon (Elia, 1994; O’Conor, 1994). School environments are expected to be safe, positive, and nurturing, supporting the growth and development of all youth. Despite this common expectation, sexual minority youth may frequently experience stigmatization and victimization. This may include a substantial number of stressors, such as physical or verbal homophobic bullying and rejection by both teachers and peers, as well as cyberbullying and discrimination (Jordan et al., 1997). A study by Remafedi (1987) found that due to bullying $80 \%$ of participants showed declining performance in school, $40 \%$ reported absence, $30 \%$ dropped out, and 40\% lost friends. Similarly, Marsiglio (1993) concluded that $88 \%$ of heterosexual males in high school reported finding gay relationships repulsive, and only $12 \%$ felt they could be friends with a gay male, which, in sexual minorities, may lead to feelings of rejection and lowered selfesteem. As a significant and negative outcome of heterosexism in the school environment, bullying often becomes a central issue to the existence of sexual minority youth. In general, actual or perceived bullying may lead to peer rejection, isolation, and a sharp decrease in self-esteem (O'Moore \& Kirkham, 2001; Overbeek et al., 2010). More relevantly to sexual minority youth, homophobic bullying has also been shown to be a significant factor in declines in self-esteem (Waldo et al., 1998; Russell et al., 2011). 


\section{Family and friends}

In an early research in studies on the influence of the family environment on sexual minorities, Savin-Williams (1989) examined a sample of 317 youth using self-report measures and found that for girls a more satisfying relationship with their mother predicted higher self-esteem. In addition, for boys, satisfying relationship with both parents predicted higher selfesteem. To further explore this idea, in their study of 72 lesbian, gay, and bisexual youth, Floyd et al. (1999) found that the more positive parents' attitudes were to their child sexual orientation, the higher youth scored on self-esteem measures. Similarly, Legate et al. (2012) found that sexual minority participants who were out to most family and friends presented higher self-esteem, which was further augmented by the amount of perceived social support. Underpinning this finding, Kosciw et al. (2012) reported, after examining a sample of sexual minority high school students, that being more out to one's environment was significantly related to higher levels of self-esteem. Recent evidence from Jackson and Mohr (2016) is in line with the body of these findings, suggesting that there is a significant association between concealing one's sexual orientation and higher levels of self-stigma, lower life satisfaction, as well as lower levels of identity strength. Contradictory to the supporting evidence, other studies (Russell \& Joyner, 2001; Diamond \& Lucas, 2004) have found that although sexual minority youth may have positive, accepting, and nurturing environments, they may still present lower self-esteem because of internalized issues with peer group belonging.

\section{Community}

Social isolation of sexual minority youth in the community setting was pinpointed by Hetrick and Martin (1987) as an outcome of rejection and stigmatization they so often must face. Reports on these issues were supported by evidence from Berger (1992) who found self-esteem to have a moderate to strong correlation with supportiveness in a sample of gay men. This was further evidenced by Savin-Williams (1994) when examining a sample of gay and lesbian youth, showing that a negative social context, such as a homophobic culture of verbal abuse, threats, and physical harm, had a negative correlation with internalized homophobia and self-esteem. Considering this evidence, the presence and availability of sexual minority youth support organizations, support groups, and the school presence of gay-straight alliances (GSAs) in schools seems especially important, because they not only support the individual, but also provide social connectedness with others and a sense of belonging (Berkman, 1995). This was supported by evidence from research conducted by Lee and Robbins
(1998) on 185 students, showing that social connectedness is related to enhanced levels of self-esteem. Further support was provided by Detrie and Lease (2007) who examined a sample of 218 lesbian, gay, and bisexual youth, reporting a positive association between social connectedness and self-esteem. The importance of positive social ties was also underpinned by evidence from recent studies examining the influence of GSAs (Toomey et al., 2011; Poteat et al., 2015; Ioverno et al., 2016). They found that GSA presence, increased GSA participation, and perceived social support from GSAs were associated with increased self-esteem and a higher tendency to come out to friends, family, and peers.

\section{Religiosity}

As part of the social context, a religious context may act as an origin of self-conflict for gay and lesbian youth. In one of the earliest studies in this field, Clingman and Fowler (1976) examined a sample of gay men at the Gay Metropolitan Church and found that church attendees who experienced belonging and positive religious experiences also reported higher selfesteem, as opposed to those gay men who did not attend this church. In a study of 66 gay, lesbian, and bisexual adults, Shuck and Liddle (2001) found that over $66 \%$ of participants reported a friction between their sexual and religious identities. Continuing to research the religious experiences of sexual minorities, Sherkat (2002) reported that of the 2500 religious movements in the United States, almost all disapprove of nonheterosexual orientations, which leads to a frustrating, judgmental, and conflicting social context for sexual minorities. Following their footsteps, in a recent research, Dahl and Galliher (2010) examined 106 sexual minority youth looking for correlations between religiosity and self-esteem. Like previous studies, their findings also suggested that religious experiences initiating positive affect (such as kind and forgiving God) are associated with increased self-esteem, whereas negative religious experiences (such as fear of God, fear of judgment) were associated with low self-evaluation, low self-worth, and decreased self-esteem.

\section{Assessing social context}

In an early study, Thoits (1985) coined the idea that socialization during adolescence and young adulthood inevitably exposes sexual minority youth to the negative attitudes that society commonly holds against nonheterosexual individuals. This learning process often leads to labeling of oneself as "homosexual" with a negative connotation, deviant from the "majority," leading to directing the social homophobia toward the self, resulting in internalized homophobia. Seeing the social 
context as hostile may lead to maladaptive responses, decreased self-esteem, and aggression toward oneself and toward other sexual minority individuals (Garnets et al., 1990; Herek et al., 2009; Frost \& Meyer, 2009; Szymanski \& Gupta, 2009).

Evidence for these views came from Meyer (1995) who studied the differences between gay and straight men, suggesting that gay men overall show higher distress in areas such as selfacceptance and alienation. In a sample of 741 gay men, he found that internalized homophobia, stigma, and victimization predicted higher distress. In addition, more frequent stigmatization was also related to lowered self-esteem and distorted self-perception because of internalized homophobia. Further supporting evidence was provided by Riggle et al. (2017) who studied a sample of 373 lesbian, gay, and bisexual participants and found that increased perception of stigma was related to higher distress and perception of contextual hostility and also to lower levels of self-esteem. These conclusions also suggest that social context of and attitudes toward sexual minority youth may have considerable consequences when trying to develop a healthy self-concept (Pachankis, 2007).

Further assessing the influence of the social environment, Herek et al. (2009) examined a community sample of 2259 lesbian, gay, and bisexual individuals. The study reported that higher levels of internalized homophobia led to lower levels of self-esteem.

\section{Aims of this review}

Abundant research has been carried out on the factors positively or negatively impacting on the well-being of sexual minority youth, addressing mainly the issues of their relationships with heterosexual peers or the issues of sexual risk taking and sexually transmitted diseases. Although other aspects, such as social context and its effect on the association between self-concept and well-being of sexual minorities, have been researched, the literature in this area is relatively new, and findings are not homogeneous.

At present, no review has been conducted using the existing literature to synthesize findings on the relationship of the two concepts: social context and self-concept. Thus, the aim of this systematic review is to assess the association between social context and self-concept in relation to sexual minority youth. The secondary aim of this systematic review is to synthesize the findings of current research studies and provide implications for the future for both research and practice, with a goal to assist relevant organizations and professionals in helping sexual minority youth achieve higher self-esteem, a healthy self-concept, and overall well-being. The following research questions were set:

What is the relationship between social context and selfconcept in gay and lesbian youth?

What potential methodological threats are present in the literature?

Based on the research questions, the following were predicted:

A more positive social context would be associated with a more positive self-concept.

The reviewed literature would present methodological inconsistencies.

\section{METHOD}

\section{Inclusion/exclusion criteria}

Journal articles were required to meet the following criteria in order to be included in the present review. These criteria were defined in accordance to the SPiDER requirements: sample, phenomenon of interest, design, evaluation outcomes, and research type (Murdoch University, 2019). Therefore regarding the inclusion criteria for the review, the papers had to include gay and lesbian young people only (aged between 12 and 25), the articles had to include a measure of social context, both cross-sectional and longitudinal designs were included, the articles had to include a measure of self-concept as an outcome, and research had to be quantitative. Further to that, all studies also had to be written in English and no study could include a mixed sample of heterosexual and LGBT participants.

\section{Search strategy}

The present review followed PRISMA guidelines (British Medical Journal, 2009) in conducting the literature search, which was performed in online databases accessible through the University of Edinburgh online library. The databases are representative of the literature published in the research area examined by this review. Because of accessibility reasons, databases were searched in two groups. First, search was conducted in the group involving Academic Search Complete, Child Development \& Adolescent Studies, ERIC, Humanities International Complete, and Sociology Course Ultimate. Second, search was conducted in the group involving PsycINFO, PsycARTICLES, AMED, and Global Health. All searches were conducted in July 2017. 


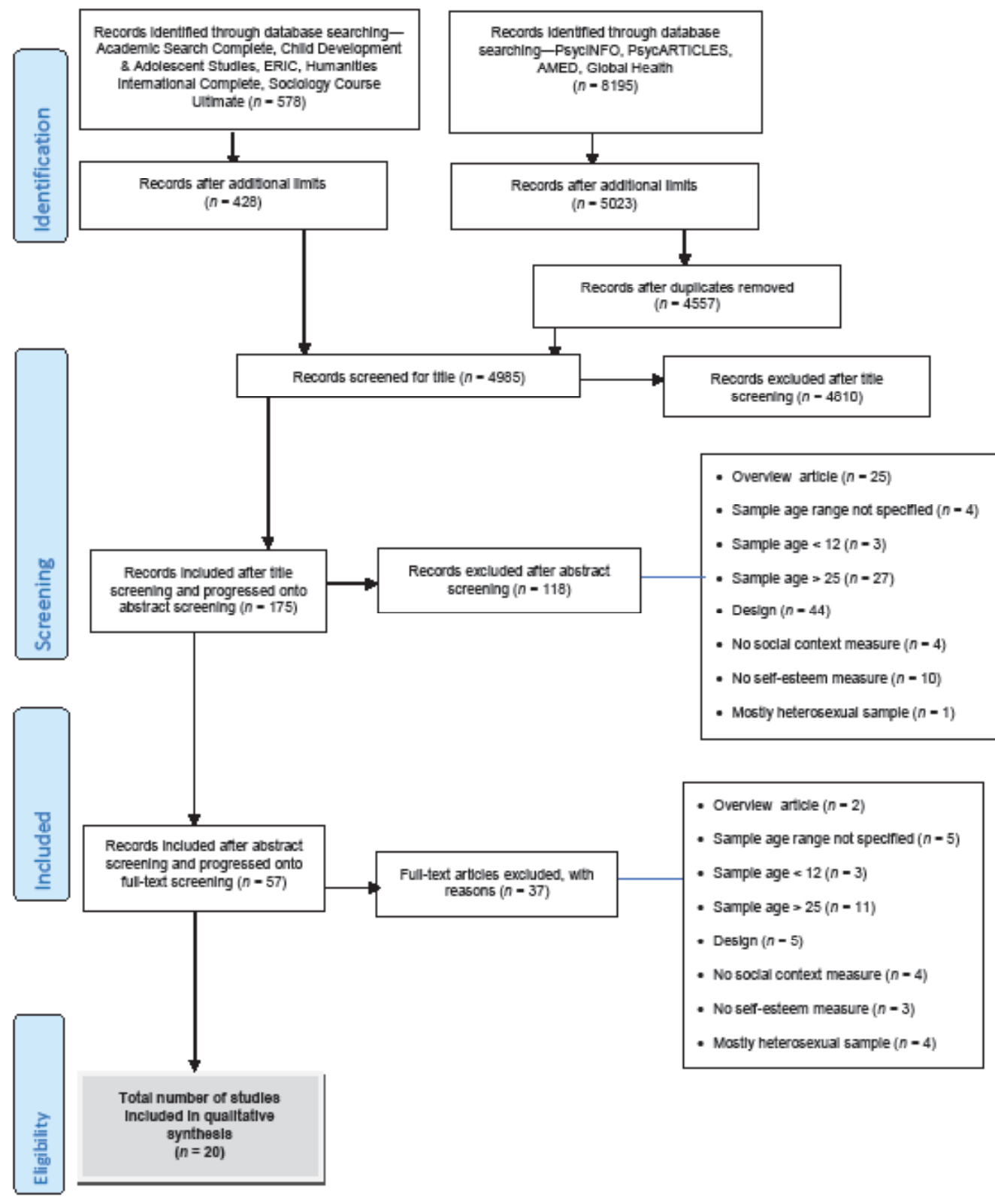

Figure 1. PRISMA flow diagram of the literature search process.

The following search terms were used: self-concept OR selfesteem AND gay OR lesbian OR bisexual OR sexual minority OR non-heterosexual AND youth OR adolescen* OR student. The initial results produced were narrowed by the criteria "peer reviewed journal only." Range on publication date was not set. This decision was made based on the relatively young nature of research in the area, and because of the lack of reviews combining gay and lesbian youth, and self-concept and social context.

All searches and initial selection of articles were conducted by FH with NF also reviewing the inclusion/exclusion criteria for selected abstracts. Agreement on articles retained was 100\%. The process of article selection is illustrated in Figure 1. This systematic review was conducted without financial or other support.

\section{Quality assessment}

Quality assessment of all included articles was performed with the use of an adapted version of the Strengthening the Reporting of Observational Studies in Epidemiology (STROBE) checklist (Von Elm et al. 2007). The STROBE checklist appraises 
Table 1. Quality ratings of studies

\begin{tabular}{|c|c|c|c|c|c|c|c|c|c|c|c|c|c|c|c|c|c|c|c|c|}
\hline \multirow{2}{*}{\begin{tabular}{|l} 
\\
Number
\end{tabular}} & \multirow[b]{2}{*}{$\begin{array}{l}\text { Author } \\
\text { (Year) }\end{array}$} & \multirow[b]{2}{*}{$\begin{array}{l}\text { Title/ } \\
\text { Abstract }\end{array}$} & \multicolumn{4}{|c|}{ Introduction } & \multicolumn{14}{|c|}{ Methods } \\
\hline & & & \multicolumn{2}{|c|}{\begin{tabular}{|l} 
Background \\
Rationale
\end{tabular}} & \multicolumn{2}{|c|}{ Objectives } & \begin{tabular}{|l|} 
Study \\
Design
\end{tabular} & Settin & \multicolumn{2}{|c|}{ g Participants } & \multicolumn{2}{|c|}{ Variables } & \multicolumn{2}{|c|}{$\begin{array}{l}\text { Data Sources/ } \\
\text { Measurement }\end{array}$} & Bias & $\begin{array}{l}\text { Study } \\
\text { Size }\end{array}$ & \multicolumn{2}{|c|}{$\begin{array}{l}\text { Quantitative } \\
\text { Variables }\end{array}$} & \multicolumn{2}{|c|}{$\begin{array}{l}\text { Statistical } \\
\text { Methods }\end{array}$} \\
\hline 1 & \begin{tabular}{|l} 
Detrie \& \\
Lease (2007)
\end{tabular} & 1 & 2 & & 2 & & 0 & 2 & 2 & & 2 & & 2 & & 1 & 2 & 1 & & 1 & \\
\hline 2 & \begin{tabular}{|l|} 
Dahl \& \\
Galliher \\
$(2010)$ \\
\end{tabular} & 1 & 2 & & 1 & & 0 & 1 & 2 & & 0 & & 1 & & 0 & 1 & 1 & & 2 & \\
\hline 3 & \begin{tabular}{|l|} 
Grossman \& \\
Kerner (1998)
\end{tabular} & 1 & 2 & & 2 & & 1 & 2 & 2 & & 2 & & 2 & & 0 & 1 & 2 & & 1 & \\
\hline 4 & \begin{tabular}{|l|} 
loverno et al. \\
$(2016)$
\end{tabular} & 2 & 2 & & 2 & & 2 & 2 & 2 & & 2 & & 2 & & 1 & 2 & 2 & & 2 & \\
\hline 5 & \begin{tabular}{|l|} 
Poteat et al. \\
$(2015)$
\end{tabular} & 1 & 2 & & 2 & & 1 & 2 & 2 & & 1 & & 2 & & 1 & 2 & 2 & & 1 & \\
\hline 6 & $\begin{array}{l}\text { Hershberger } \\
\text { \& D’Augelli } \\
\text { (1995) }\end{array}$ & 2 & 2 & & 1 & & 2 & 2 & 2 & & 2 & & 2 & & 0 & 2 & 2 & & 2 & \\
\hline 7 & $\begin{array}{l}\text { Savin- } \\
\text { Williams } \\
\text { (1989) }\end{array}$ & 1 & 1 & & 1 & & 1 & 2 & 2 & & 2 & & 2 & & 1 & 2 & 2 & & 1 & \\
\hline 8 & $\begin{array}{l}\text { Snapp et al. } \\
(2015)\end{array}$ & 2 & 2 & & 2 & & 2 & 2 & 2 & & 1 & & 2 & & 0 & 2 & 2 & & 1 & \\
\hline 9 & $\begin{array}{l}\text { Woodford et } \\
\text { al. (2015) }\end{array}$ & 1 & 2 & & 1 & & 0 & 1 & 1 & & 1 & & 2 & & 2 & 0 & 2 & & 1 & \\
\hline 10 & $\begin{array}{l}\text { Rotheram- } \\
\text { Borus et al. } \\
\text { (1995) }\end{array}$ & 1 & 2 & & 1 & & 1 & 2 & 2 & & 2 & & 2 & & 2 & 2 & 2 & & 1 & \\
\hline 11 & \begin{tabular}{|l|} 
D'Augelli \& \\
Hershberger \\
(1993) \\
\end{tabular} & 1 & 2 & & 0 & & 0 & 2 & 2 & & 1 & & 2 & & 1 & 2 & 1 & & 2 & \\
\hline 12 & \begin{tabular}{|l|} 
Russel et al. \\
$(2014)$
\end{tabular} & 1 & 2 & & 2 & & 1 & 1 & 1 & & 2 & & 2 & & 0 & 1 & 2 & & 1 & \\
\hline 13 & $\begin{array}{l}\text { Rosario et al. } \\
(2005)\end{array}$ & 2 & 2 & & 2 & & 1 & 2 & 2 & & 1 & & 2 & & 0 & 2 & 2 & & 2 & \\
\hline 14 & \begin{tabular}{|l|} 
Blais et al. \\
$(2014)$
\end{tabular} & 1 & 2 & & 2 & & 1 & 2 & 2 & & 1 & & 2 & & 2 & 2 & 2 & & 2 & \\
\hline 15 & \begin{tabular}{|l|}
$\begin{array}{l}\text { Wilkerson et } \\
\text { al. (2017) }\end{array}$ \\
\end{tabular} & 2 & 2 & & 1 & & 1 & 0 & 1 & & 2 & & 2 & & 1 & 0 & 2 & & 2 & \\
\hline 16 & \begin{tabular}{|l|} 
Vincke \& van \\
Heeringen \\
$(2002)$
\end{tabular} & 2 & 1 & & 2 & & 2 & 2 & 2 & & 2 & & 2 & & 2 & 2 & 2 & & 2 & \\
\hline 17 & \begin{tabular}{|l|}
$\begin{array}{l}\text { Bauermeister } \\
\text { et al. (2010) }\end{array}$ \\
\end{tabular} & 2 & 2 & & 2 & & 2 & 2 & 1 & & 2 & & 2 & & 2 & 2 & 2 & & 2 & \\
\hline 18 & \begin{tabular}{|l|} 
Rosario et al. \\
(2011)
\end{tabular} & 2 & 2 & & 2 & & 2 & 2 & 2 & & 2 & & 2 & & 2 & 1 & 2 & & 1 & \\
\hline 19 & \begin{tabular}{|l|} 
Bos et al. \\
$(2008)$
\end{tabular} & 2 & 2 & & 2 & & 2 & 2 & 2 & & 1 & & 1 & & 0 & 2 & 2 & & 2 & \\
\hline 20 & \begin{tabular}{|l|}
$\begin{array}{l}\text { Toomey et al. } \\
\text { (2011) }\end{array}$ \\
\end{tabular} & 2 & 2 & & 2 & & 2 & 2 & 2 & & 2 & & 2 & & 2 & 2 & 2 & & 2 & \\
\hline & & Results & & & & & & & & Disc & cussic & & & & & & & & & \\
\hline Number & \begin{tabular}{|l|} 
Author \\
(Year)
\end{tabular} & Participar & & \begin{tabular}{|l} 
Descript \\
Data
\end{tabular} & & $\begin{array}{l}\text { Outcome } \\
\text { Data }\end{array}$ & \begin{tabular}{l|l} 
ie & $\begin{array}{l}\text { Mai } \\
\text { Res }\end{array}$ \\
\end{tabular} & & $\begin{array}{l}\text { Dther } \\
\text { Analysis }\end{array}$ & $\begin{array}{l}\text { Key } \\
\text { Rest }\end{array}$ & ults & $\begin{array}{l}\text { Lim } \\
\text { tior }\end{array}$ & $\begin{array}{l}\text { nita- } \\
\text { ns }\end{array}$ & Interpreta & ation & Genera & alizability $F$ & Fund & ding & \begin{tabular}{|l|} 
Total \\
Score
\end{tabular} \\
\hline 1 & \begin{tabular}{|l|} 
Detrie \& \\
Lease (2007) \\
\end{tabular} & 2 & & 1 & & 1 & 1 & 1 & & 2 & & 2 & & 2 & & 2 & & 0 & & 32 \\
\hline 2 & \begin{tabular}{|l|} 
Dahl \& \\
Galliher \\
(2010) \\
\end{tabular} & 0 & & 2 & & 2 & 1 & 2 & 2 & 2 & & 2 & & 2 & & 2 & & 0 & & 27 \\
\hline 3 & \begin{tabular}{|l|} 
Grossman \& \\
Kerner (1998) \\
\end{tabular} & 0 & & 1 & & 2 & 2 & 1 & & 2 & & 1 & & 2 & & 2 & & 0 & & 31 \\
\hline 4 & $\begin{array}{l}\text { loverno et al. } \\
\text { (2016) }\end{array}$ & 2 & & 2 & & 2 & 2 & 2 & & 2 & & 2 & & 2 & & 2 & & 2 & & 43 \\
\hline 5 & $\begin{array}{l}\text { Poteat et al. } \\
\text { (2015) }\end{array}$ & 0 & & 1 & & 2 & 1 & 2 & & 2 & & 2 & & 2 & & 1 & & 2 & & 34 \\
\hline
\end{tabular}


Table 1. Quality ratings of studies

\begin{tabular}{|c|c|c|c|c|c|c|c|c|c|c|c|c|}
\hline \multirow[b]{2}{*}{ Number } & \multirow[b]{2}{*}{$\begin{array}{l}\text { Author } \\
\text { (Year) }\end{array}$} & \multicolumn{5}{|l|}{ Results } & \multicolumn{5}{|c|}{ Discussion } & \multirow[b]{2}{*}{\begin{tabular}{|l|} 
Total \\
Score
\end{tabular}} \\
\hline & & Participants & $\begin{array}{l}\text { Descriptive } \\
\text { Data }\end{array}$ & $\begin{array}{l}\text { Outcome } \\
\text { Data }\end{array}$ & \begin{tabular}{|l|} 
Main \\
Results
\end{tabular} & $\begin{array}{l}\text { Other } \\
\text { Analysis }\end{array}$ & \begin{tabular}{|l|} 
Key \\
Results
\end{tabular} & $\begin{array}{l}\text { Limita- } \\
\text { tions }\end{array}$ & Interpretation & Generalizability & Funding & \\
\hline 6 & $\begin{array}{l}\text { Hershberger } \\
\text { \& D'Augelli } \\
\text { (1995) }\end{array}$ & 1 & 2 & 2 & 1 & 2 & 2 & 2 & 2 & 2 & 2 & 39 \\
\hline 7 & \begin{tabular}{|l|} 
Savin- \\
Williams \\
(1989)
\end{tabular} & 0 & 1 & 2 & 1 & 1 & 2 & 2 & 2 & 1 & 0 & 30 \\
\hline 8 & $\begin{array}{l}\text { Snapp et al. } \\
\text { (2015) }\end{array}$ & 0 & 1 & 2 & 1 & 1 & 2 & 2 & 2 & 2 & 2 & 35 \\
\hline 9 & $\begin{array}{l}\text { Woodford et } \\
\text { al. (2015) }\end{array}$ & 0 & 1 & 1 & 1 & 1 & 2 & 2 & 2 & 2 & 2 & 28 \\
\hline 10 & $\begin{array}{l}\text { Rotheram- } \\
\text { Borus et al. } \\
\text { (1995) }\end{array}$ & 1 & 1 & 2 & 1 & 2 & 2 & 1 & 2 & 2 & 2 & 36 \\
\hline 11 & \begin{tabular}{|l|} 
D’Augelli \& \\
Hershberger \\
(1993)
\end{tabular} & 2 & 1 & 2 & 1 & 2 & 2 & 1 & 1 & 2 & 2 & 32 \\
\hline 12 & $\begin{array}{l}\text { Russel et al. } \\
(2014)\end{array}$ & 0 & 0 & 2 & 2 & 2 & 2 & 2 & 2 & 2 & 0 & 30 \\
\hline 13 & $\begin{array}{l}\text { Rosario et al. } \\
(2005)\end{array}$ & 0 & 2 & 2 & 1 & 2 & 2 & 2 & 2 & 2 & 2 & 37 \\
\hline 14 & \begin{tabular}{|l|} 
Blais et al. \\
$(2014)$
\end{tabular} & 0 & 2 & 2 & 2 & 2 & 2 & 2 & 2 & 2 & 2 & 39 \\
\hline 15 & $\begin{array}{l}\text { Wilkerson et } \\
\text { al. (2017) }\end{array}$ & 0 & 2 & 2 & 2 & 2 & 1 & 1 & 1 & 0 & 2 & 29 \\
\hline 16 & $\begin{array}{l}\text { Vincke \& van } \\
\text { Heeringen } \\
(2002) \\
\end{array}$ & 2 & 2 & 2 & 2 & 2 & 2 & 2 & 2 & 2 & 2 & 43 \\
\hline 17 & $\begin{array}{l}\text { Bauermeister } \\
\text { et al. }(2010)\end{array}$ & 2 & 2 & 2 & 1 & 1 & 1 & 2 & 2 & 2 & 2 & 40 \\
\hline 18 & $\begin{array}{l}\text { Rosario et al. } \\
\text { (2011) }\end{array}$ & 1 & 1 & 2 & 1 & 2 & 2 & 2 & 2 & 2 & 2 & 39 \\
\hline 19 & \begin{tabular}{|l|} 
Bos et al. \\
$(2008)$
\end{tabular} & 0 & 2 & 2 & 1 & 2 & 2 & 2 & 2 & 2 & 2 & 37 \\
\hline 20 & \begin{tabular}{|l} 
Toomey et al. \\
(2011)
\end{tabular} & 0 & 2 & 2 & 2 & 2 & 2 & 2 & 2 & 2 & 2 & 42 \\
\hline
\end{tabular}

methodological quality of cohort, cross-sectional, and casecontrol studies. In the present review, it was used to assess the mostly cross-sectional and the few longitudinal cohort studies. The checklist evaluates papers based on a 22 -item list of criteria. Possible ratings for each criterion are as follows: "Well-covered" ( 2 points), "Adequately covered" (1 point), "Not covered" (0 point), and "Not applicable" (0 point). The total number of points for one study can range between 0 and 44 .

\section{RESULTS}

\section{Search results}

Combined primary search yielded 5451 papers. After duplicates were removed, 4985 articles remained, of which 4810 were excluded after title review. Abstracts of the remaining 175 articles were screened, and for reasons explained in Figure 1, 118 were further eliminated. Fifty-seven articles were assessed by examining their full text using the inclusion/exclusion criteria, yielding a final 20 articles to be included in the qualitative synthesis of the present review. The quality, descriptive, and methodological characteristics of the studies will be examined before discussing the findings of the studies.

\section{Quality of the studies}

As can be seen in Table 1, 11 of the 20 studies scored in the upper quartile ( $>33)$, indicating that more than half the studies were of good to excellent methodological quality. Of those that scored lower, they all still fulfilled more than half of the methodological criteria (all scores above 22).

\section{Descriptive characteristics of studies}

Table 2 represents a summary of the main descriptive features of the chosen journal articles. 
Table 2. Descriptive characteristics of studies

\begin{tabular}{|c|c|c|c|c|c|c|c|c|}
\hline & $\begin{array}{l}\text { Author (Year) } \\
\text { Country }\end{array}$ & Design & $\begin{array}{l}\text { Sample size }(\mathrm{N}) / \\
\text { Age Range/Sexual } \\
\text { Orientation }\end{array}$ & $\begin{array}{l}\text { Sexual Orientation } \\
\text { Measure }\end{array}$ & Social Context Measure & $\begin{array}{l}\text { Self-Concept } \\
\text { Measure }\end{array}$ & Level of Analysis & Threats \\
\hline 1 & $\begin{array}{l}\text { Detrie \& Lease } \\
\text { (2007) } \\
\text { USA }\end{array}$ & CS & $\begin{array}{l}N=218 \\
\text { Age }=14-22 \\
L, G, B, O N H\end{array}$ & PIQ & $\begin{array}{l}\text { PSS-Fa; } \\
\text { PSS-Fr; } \\
\text { SCS; } \\
\text { PWB }\end{array}$ & \begin{tabular}{|l|} 
CSES; \\
PWBS (self- \\
acceptance)
\end{tabular} & $\begin{array}{l}\text { Individual } \\
\text { (online survey) }\end{array}$ & $\begin{array}{l}\text { Sample demographics } \\
\text { (Caucasian, middle to } \\
\text { upper class) } \\
\text { Participants already } \\
\text { out } \\
\text { Correlational design } \\
\text { (no causal inferences) } \\
\text { Self-report measures }\end{array}$ \\
\hline 2 & $\begin{array}{l}\text { Dahl \& Galliher } \\
\text { (2010) } \\
\text { USA }\end{array}$ & CS & $\begin{array}{l}N=106 \\
\text { Age }=18-24 \\
\text { L, G, B, ONH }\end{array}$ & $\begin{array}{l}\text { Demographic } \\
\text { information/sexual } \\
\text { orientation history }\end{array}$ & $\begin{array}{l}\text { MOGS; } \\
\text { CES-DS; } \\
\text { RCSS; } \\
\text { BMMRS }\end{array}$ & RSES & $\begin{array}{l}\text { Individual } \\
\text { (online survey) }\end{array}$ & $\begin{array}{l}\text { Small sample size } \\
\text { Convenience sampling } \\
\text { Self-report measures }\end{array}$ \\
\hline 3 & $\begin{array}{l}\text { Grossman \& } \\
\text { Kerner (1998) } \\
\text { USA }\end{array}$ & CS & $\begin{array}{l}N=90 \\
\text { Age }=14-21 \\
\text { L, G }\end{array}$ & $\begin{array}{l}\text { Sociodemographic } \\
\text { and risk factor } \\
\text { questionnaire }\end{array}$ & SNS & RSES & $\begin{array}{l}\text { Individual } \\
\text { (in person) }\end{array}$ & $\begin{array}{l}\text { Small sample size } \\
\text { Convenience sample } \\
\text { Geographically limited } \\
\text { urban sample } \\
\text { Caucasians } \\
\text { underrepresented } \\
\text { Population not } \\
\text { representative } \\
\text { Self-report measure } \\
\text { Issues with } \\
\text { generalizability and } \\
\text { external validity }\end{array}$ \\
\hline 4 & $\begin{array}{l}\text { loverno et al. } \\
\text { (2016) } \\
\text { USA }\end{array}$ & Long & $\begin{array}{l}N=327 \\
\text { Age }=15-21 \\
L, G, B, Q\end{array}$ & $\begin{array}{l}\text { Demographic } \\
\text { information }\end{array}$ & $\begin{array}{l}\text { GSA presence and } \\
\text { participation; } \\
\text { Homophobic bullying } \\
\text { (single-item question } \\
\text { 5-point); } \\
\text { School safety (4-point } \\
\text { single question) }\end{array}$ & RSES & $\begin{array}{l}\text { Individual } \\
\text { (questionnaire-in } \\
\text { person or online) }\end{array}$ & $\begin{array}{l}\text { Small sample size } \\
\text { Single item measure } \\
\text { used for two key } \\
\text { indicators } \\
\text { Geographically } \\
\text { limited sample (limits } \\
\text { generalizability) }\end{array}$ \\
\hline 5 & $\begin{array}{l}\text { Poteat et al. } \\
\text { (2015) } \\
\text { USA }\end{array}$ & CS & $\begin{array}{l}N=146 \\
\text { Age }=14-19 \\
L, G, B, Q, O N H\end{array}$ & $\begin{array}{l}\text { Demographic } \\
\text { information }\end{array}$ & $\begin{array}{l}\text { Victimization (verbal, } \\
\text { relational, and physical); } \\
\text { GSA Support and } \\
\text { Advocacy; } \\
\text { NRI }\end{array}$ & RSES & $\begin{array}{l}\text { Individual } \\
\text { (in person) }\end{array}$ & $\begin{array}{l}\text { No causal inferences } \\
\text { Population not } \\
\text { representative } \\
\text { Sample geographically } \\
\text { limited (issues with } \\
\text { generalizability) } \\
\text { Small sample size } \\
\text { Self-report measures }\end{array}$ \\
\hline 6 & $\begin{array}{l}\text { Hershberger } \\
\text { \& D'Augelli } \\
\text { (1995) } \\
\text { USA }\end{array}$ & CS & $\begin{array}{l}N=165 \text { (22 omitted- } \\
\text { unspecified sex, } \\
\text { or identified as } \\
\text { heterosexual, or age > 21) } \\
(+34 \text { omitted - missing } \\
\text { data) } \\
\text { Age }=15-21 \\
\text { L, G, B }\end{array}$ & $\begin{array}{l}\text { Sampling done is } \\
\text { lesbian and gay } \\
\text { community centers }\end{array}$ & $\begin{array}{l}\text { Victimization variables } \\
\text { (Attack I, II, III } \\
\text { representing escalating } \\
\text { levels of violence- } \\
\text { frequency to be assessed } \\
0-3 \text { ); } \\
\text { family support variables } \\
\text { (family acceptance } 1-4 \text {, } \\
\text { family protection } 0-4 \text {, } \\
\text { family relations } 0-3 \text { ); } \\
\text { BSI }\end{array}$ & $\begin{array}{l}\text { RSES; } \\
\text { single-item } \\
\text { question on } \\
\text { "Comfort } \\
\text { with sexual } \\
\text { orientation" }\end{array}$ & $\begin{array}{l}\text { Individual } \\
\text { (individual-surveys } \\
\text { mailed to youth } \\
\text { groups) }\end{array}$ & $\begin{array}{l}\text { Small sample size } \\
\text { Sample not } \\
\text { representative } \\
\text { Small number of } \\
\text { female participants } \\
\text { Self-report measures } \\
\text { Interpretation of } \\
\text { developmental } \\
\text { processes may be } \\
\text { speculative without } \\
\text { longitudinal design }\end{array}$ \\
\hline
\end{tabular}


Table 2. Descriptive characteristics of studies

\begin{tabular}{|c|c|c|c|c|c|c|c|c|}
\hline & $\begin{array}{l}\text { Author (Year) } \\
\text { Country }\end{array}$ & Design & $\begin{array}{l}\text { Sample size }(N) / \\
\text { Age Range/Sexual } \\
\text { Orientation }\end{array}$ & $\begin{array}{l}\text { Sexual Orientation } \\
\text { Measure }\end{array}$ & Social Context Measure & $\begin{array}{l}\text { Self-Concept } \\
\text { Measure }\end{array}$ & Level of Analysis & Threats \\
\hline 7 & $\begin{array}{l}\text { Savin-Williams } \\
\text { (1989) } \\
\text { USA }\end{array}$ & CS & $\begin{array}{l}N=317 \\
\text { Age }=14-23 \\
L, G\end{array}$ & GAL-Q & $\begin{array}{l}\text { GAL-Q (parental } \\
\text { knowledge of their } \\
\text { child's homosexuality; } \\
\text { satisfaction with maternal } \\
\text { and paternal relationship; } \\
\text { contact with parents) }\end{array}$ & RSES & $\begin{array}{l}\text { Individual } \\
\text { (surveys) }\end{array}$ & $\begin{array}{l}\text { No causal inferences } \\
\text { Small number of } \\
\text { female participants } \\
\text { Convenience and } \\
\text { snowball sampling } \\
\text { (issues with } \\
\text { generalizability) } \\
\text { Ethnic minorities } \\
\text { underrepresented } \\
\text { Low education } \\
\text { individuals } \\
\text { underrepresented }\end{array}$ \\
\hline 8 & $\begin{array}{l}\text { Snapp et al. } \\
\text { (2015) } \\
\text { USA, Canada }\end{array}$ & CS & $\begin{array}{l}N=245 \\
\text { Age }=21-25 \\
L, G, B, T\end{array}$ & $\begin{array}{l}\text { Personal } \\
\text { characteristics } \\
\text { questionnaire }\end{array}$ & $\begin{array}{l}\text { FAS; Retrospective } \\
\text { ESM (friend support; } \\
\text { community support; } \\
\text { young adult adjustment } \\
\text { and well-being; life } \\
\text { situation) }\end{array}$ & $\begin{array}{l}\text { RSES; } \\
\text { LGBT-SES }\end{array}$ & Individual & $\begin{array}{l}\text { ESM is subjective } \\
\text { Self-report measures } \\
\text { Convenience sampling } \\
\text { Geographically } \\
\text { limited (issues with } \\
\text { generalizability) } \\
\text { Retrospective cross- } \\
\text { sectional design } \\
\text { (recall bias) } \\
\text { No causal inferences }\end{array}$ \\
\hline 9 & $\begin{array}{l}\text { Woodford et al. } \\
\text { (2015) } \\
\text { Canada, USA }\end{array}$ & CS & $\begin{array}{l}N=187 \\
\text { Age }=18-25 \\
\text { L, G, B, Q, ONH }\end{array}$ & $\begin{array}{l}\text { Two single-item } \\
\text { measures on sexual } \\
\text { orientation and } \\
\text { gender identity/ } \\
\text { LGBQ identity } \\
\text { salience lone-item } \\
\text { question-4-point } \\
\text { scale) }\end{array}$ & $\begin{array}{l}\text { Microaggressions (distal/ } \\
\text { proximal); } \\
\text { GAD }\end{array}$ & RSES & $\begin{array}{l}\text { Individual } \\
\text { (anonymous web- } \\
\text { based survey) }\end{array}$ & $\begin{array}{l}\text { No causal inferences } \\
\text { Small sample size } \\
\text { Whites } \\
\text { overrepresented } \\
\text { Self-report measures. } \\
\text { Convenience } \\
\text { sampling. Internal } \\
\text { consistency of self- } \\
\text { developed scales may } \\
\text { be questionable }\end{array}$ \\
\hline 10 & $\begin{array}{l}\text { Rotheram- } \\
\text { Borus et al. } \\
\text { (1995] } \\
\text { USA }\end{array}$ & Long & $\begin{array}{l}N=136 \text { (+5 } \\
\text { omitted-identified } \\
\text { as heterosexual) (+6 } \\
\text { withdrawn) } \\
\text { Age = 14-19 } \\
\text { G, B, Q }\end{array}$ & $\begin{array}{l}\text { Sampling done at } \\
\text { LGBT drop-in center }\end{array}$ & $\begin{array}{l}\text { Gay-related events } \\
\text { (7-item measure } \\
\text { constructed by authors); } \\
\text { LEC }\end{array}$ & RSES & Individual & $\begin{array}{l}\text { Small sample size } \\
\text { Convenience sample } \\
\text { Exclusively male } \\
\text { sample lissues with } \\
\text { generalizability) }\end{array}$ \\
\hline 11 & $\begin{array}{l}\text { D’Augelli \& } \\
\text { Hershberger } \\
\text { (1993) } \\
\text { USA }\end{array}$ & CS & $\begin{array}{l}N=194(+27 \text { omitted }) \\
\text { Age }=15-21 \\
\text { L, G, B }\end{array}$ & $\begin{array}{l}\text { Questions on sexual } \\
\text { orientation }\end{array}$ & $\begin{array}{l}\text { Social Aspects of Sexual } \\
\text { Orientation lopenness; } \\
\text { conformity; involvement; } \\
\text { disclosure-related } \\
\text { events); } \\
\text { Disclosure of Sexual } \\
\text { Orientation Within the } \\
\text { Family litems taken from } \\
\text { Savin-Williams, 1990); } \\
\text { Mental Health Problems } \\
\text { (items taken from Mapou } \\
\text { et al., 1983); } \\
\text { BSI }\end{array}$ & RSES & $\begin{array}{l}\text { Individual } \\
\text { (questionnaires- } \\
\text { filled in individually } \\
\text { during gay/lesbian } \\
\text { group meetings) }\end{array}$ & $\begin{array}{l}\text { Small sample size } \\
\text { Urban sample } \\
\text { Underrepresentation } \\
\text { of females }(27 \%) \text {. } \\
\text { Overrepresentation of } \\
\text { whites }(66 \%) \\
\text { No causal inferences }\end{array}$ \\
\hline
\end{tabular}


Table 2. Descriptive characteristics of studies

\begin{tabular}{|c|c|c|c|c|c|c|c|c|}
\hline & $\begin{array}{l}\text { Author (Year) } \\
\text { Country }\end{array}$ & Design & $\begin{array}{l}\text { Sample size }(N) / \\
\text { Age Range/Sexual } \\
\text { Orientation }\end{array}$ & $\begin{array}{l}\text { Sexual Orientation } \\
\text { Measure }\end{array}$ & Social Context Measure & $\begin{array}{l}\text { Self-Concept } \\
\text { Measure }\end{array}$ & Level of Analysis & Threats \\
\hline 12 & $\begin{array}{l}\text { Russel et al. } \\
\text { (2014) } \\
\text { USA }\end{array}$ & CS & $\begin{array}{l}N=245 \\
\text { Age }=21-25 \\
L, G, B, Q\end{array}$ & $\begin{array}{l}\text { Demographic } \\
\text { information taken } \\
\text { from Family } \\
\text { Acceptance Project } \\
\text { young adult survey }\end{array}$ & $\begin{array}{l}\text { Self-reported past LGBT } \\
\text { school victimization } \\
\text { (middle or high)-10 items } \\
\text { measured; } \\
\text { CES-D; } \\
\text { Young adult life } \\
\text { satisfaction }\end{array}$ & RSES & $\begin{array}{l}\text { Individual } \\
\text { (computer assisted/ } \\
\text { paper and pencil) }\end{array}$ & $\begin{array}{l}\text { Geographically } \\
\text { limited (issues with } \\
\text { generalizabilityl } \\
\text { Small sample size } \\
\text { No causal inferences. } \\
\text { Retrospective } \\
\text { accounts (recall bias) } \\
\text { Self-report measures }\end{array}$ \\
\hline 13 & $\begin{array}{l}\text { Rosario et al. } \\
\text { (2005) } \\
\text { USA }\end{array}$ & Long & $\begin{array}{l}N=156 \text { ( }+8 \text { omitted }) \\
\text { Age }=14-21 \\
\text { L, G, B, ONH }\end{array}$ & $\begin{array}{l}\text { Baseline interview } \\
\text { to establish sexual } \\
\text { orientation }\end{array}$ & $\begin{array}{l}\text { Suicidality (questions at } \\
\text { baseline interview); } \\
\text { BSI; } \\
\text { DSM-III-R (conduct } \\
\text { problems); } \\
\text { PSS; } \\
\text { SOS; } \\
\text { MCSDS }\end{array}$ & RSES & \begin{tabular}{|l|l} 
Individual \\
\end{tabular} & $\begin{array}{l}\text { Presuicidal } \\
\text { psychological distress } \\
\text { not controlled for. } \\
\text { Retrospective ESM } \\
\text { (recall bias) } \\
\text { Geographically } \\
\text { limited (limited } \\
\text { generalizabilityl } \\
\text { Small sample size. } \\
\text { Convenience sampling }\end{array}$ \\
\hline 14 & $\begin{array}{l}\text { Blais et al. } \\
\text { (2014) } \\
\text { Canada }\end{array}$ & CS & $\begin{array}{l}N=300 \\
\text { Age }=14-22 \\
L, G, B, Q\end{array}$ & $\begin{array}{l}\text { Demographic } \\
\text { information }\end{array}$ & $\begin{array}{l}\text { Homophobic bullying } \\
\text { lexclusion and rejection; } \\
\text { humiliation; damage } \\
\text { to reputation-5-point } \\
\text { nominal scale); } \\
\text { LGBIS (4 items in } \\
\text { internalized homophobia) }\end{array}$ & $\begin{array}{l}\text { SDQ }(4 \text { items } \\
\text { used) }\end{array}$ & $\begin{array}{l}\text { Individual } \\
\text { (self-report } \\
\text { questionnaire) }\end{array}$ & $\begin{array}{l}\text { Mainly urban sample. } \\
\text { Underrepresentation } \\
\text { of males } \\
\text { Issues with } \\
\text { representativeness } \\
\text { and generalizability } \\
\text { No causal inferences } \\
\text { Self-report measures } \\
\text { Physical bullying not } \\
\text { included }\end{array}$ \\
\hline 15 & $\begin{array}{l}\text { Wilkerson et } \\
\text { al. (2017) } \\
\text { USA, } \\
\text { Netherlands }\end{array}$ & CS & $\begin{array}{l}N=108 \\
\text { Age }=13-20 \\
L, G, B, O N H\end{array}$ & $\begin{array}{l}\text { Hatch Youth } \\
\text { (Houston Area } \\
\text { Team Coalition } \\
\text { of Homosexuals) } \\
\text { recruitment site }\end{array}$ & $\begin{array}{l}\text { Duration of Hatch Youth } \\
\text { attendance (1 item); } \\
\text { MSPSS; } \\
\text { CES-DS }\end{array}$ & $\begin{array}{l}\text { One item } \\
\text { (5-point scale): } \\
\text { "I have high } \\
\text { self-esteem" }\end{array}$ & $\begin{array}{l}\text { Individual } \\
\text { (survey) }\end{array}$ & $\begin{array}{l}\text { No causal inferences } \\
\text { Small sample size. } \\
\text { Convenience sampling }\end{array}$ \\
\hline 16 & $\begin{array}{l}\text { Vincke \& van } \\
\text { Heeringen } \\
\text { (2002) } \\
\text { Belgium }\end{array}$ & Long & $\begin{array}{l}N=197 \\
\text { Age }=15-25 \\
L, G\end{array}$ & $\begin{array}{l}\text { Demographic } \\
\text { information }\end{array}$ & $\begin{array}{l}\text { GHQ (5 items from the } \\
\text { depression subscale); } \\
\text { BHS; } \\
\text { SSS }\end{array}$ & RSES & \begin{tabular}{|l|} 
Individual \\
(questionnaire)
\end{tabular} & $\begin{array}{l}\text { Small sample size } \\
\text { Convenience sampling } \\
\text { High attrition rate } \\
(36.9 \%) \\
\text { Geographically limited } \\
\text { Weak } \\
\text { representativeness } \\
\text { and generalizability. } \\
\text { Underrepresentation } \\
\text { of females } \\
\text { Self-report measures }\end{array}$ \\
\hline 17 & $\begin{array}{l}\text { Bauermeister } \\
\text { et al. (2010) } \\
\text { USA }\end{array}$ & Long & $\begin{array}{l}N=350 \\
\text { Age }=15-19 \\
L, G, B, O N H\end{array}$ & $\begin{array}{l}\text { Kinsey Scale (Kinsey } \\
\text { et al., 1948) }\end{array}$ & $\begin{array}{l}\text { BDI-II; } \\
\text { BSI; } \\
\text { MSPSS; } \\
\text { ISID }\end{array}$ & RSES & $\begin{array}{l}\text { Individual } \\
\text { (interviews; } \\
\text { paper and pencil } \\
\text { questionnaire) }\end{array}$ & $\begin{array}{l}\text { Convenience sample } \\
\text { Urban sample } \\
\text { No clear breakdown } \\
\text { of sexual orientation } \\
\text { of participants. High } \\
\text { attrition rate (33.7\%). } \\
\text { Self-report measure. } \\
\text { Geographically limited } \\
\text { Issues with } \\
\text { representativeness } \\
\text { and generalizability }\end{array}$ \\
\hline
\end{tabular}


Table 2. Descriptive characteristics of studies

\begin{tabular}{|c|c|c|c|c|c|c|c|c|}
\hline & $\begin{array}{l}\text { Author (Year) } \\
\text { Country }\end{array}$ & Design & $\begin{array}{l}\text { Sample size }(N) / \\
\text { Age Range/Sexual } \\
\text { Orientation }\end{array}$ & $\begin{array}{l}\text { Sexual Orientation } \\
\text { Measure }\end{array}$ & Social Context Measure & $\begin{array}{l}\text { Self-Concept } \\
\text { Measure }\end{array}$ & Level of Analysis & Threats \\
\hline 18 & $\begin{array}{l}\text { Rosario et al. } \\
\text { (2011) } \\
\text { USA }\end{array}$ & Long & $\begin{array}{l}N=156 \text { ( }+8 \text { omitted-did } \\
\text { not meet eligibility } \\
\text { criteria) } \\
\text { Age }=14-21 \\
\text { L, G, B }\end{array}$ & $\begin{array}{l}\text { Demographic } \\
\text { information }\end{array}$ & $\begin{array}{l}\text { Involvement in LGB- } \\
\text { related activities (28-item } \\
\text { checklist; Rosario et al, } \\
\text { 2001); } \\
\text { NHAI; BSI; PSS-Fa; } \\
\text { PSS-Fr; SOS; GRSE; } \\
\text { MCSDS }\end{array}$ & RSES & $\begin{array}{l}\text { Individual } \\
\text { (interview; } \\
\text { questionnaire) }\end{array}$ & $\begin{array}{l}\text { Small sample size } \\
\text { Urban sample } \\
\text { Geographically } \\
\text { limited lissues with } \\
\text { generalizability). } \\
\text { Longitudinal design } \\
\text { but only short term } \\
\text { (1 year) }\end{array}$ \\
\hline 19 & $\begin{array}{l}\text { Bos et al. } \\
\text { (2008) } \\
\text { Netherlands, } \\
\text { USA }\end{array}$ & CS & $\begin{array}{l}N=866 \\
\text { Age }=13-15 \\
L, G, B\end{array}$ & $\begin{array}{l}\text { Single question on } \\
\text { same-sex attraction }\end{array}$ & $\begin{array}{l}\text { ADM; } \\
\text { EARSI; } \\
\text { MBRS (Respect } \\
\text { subscale); } \\
\text { GHQ (depression); } \\
\text { ISQ }\end{array}$ & $\begin{array}{l}\text { SPPA (Social } \\
\text { Acceptance } \\
\text { subscale); } \\
\text { RSES }\end{array}$ & Individual & $\begin{array}{l}\text { Reliance on } \\
\text { self-reports. } \\
\text { Geographically } \\
\text { limited (issues with } \\
\text { generalizabilityl } \\
\text { No causal inferences }\end{array}$ \\
\hline 20 & $\begin{array}{l}\text { Toomey et al. } \\
\text { (2011) } \\
\text { USA }\end{array}$ & CS & $\begin{array}{l}N=245 \\
\text { Age }=21-25 \\
L, G, B, T\end{array}$ & $\begin{array}{l}\text { Demographic } \\
\text { information }\end{array}$ & $\begin{array}{l}\text { GSA presence; } \\
\text { School victimization (10 } \\
\text { items adapted from the } \\
\text { California Healthy Kids } \\
\text { Survey, 2010); } \\
\text { CES-D }\end{array}$ & RSES & $\begin{array}{l}\text { Individual } \\
\text { (computer assisted/ } \\
\text { paper and pencil) }\end{array}$ & $\begin{array}{l}\text { Small sample size. } \\
\text { Geographically } \\
\text { limited lissues with } \\
\text { generalizability) } \\
\text { No causal inferences. } \\
\text { Retrospective design } \\
\text { (recall bias) } \\
\text { Self-report measures }\end{array}$ \\
\hline
\end{tabular}

Long, longitudinal design; CS, cross-sectional design; L, lesbian; G, gay; B, bisexual; T, transgender; Q, questioning/queer; ONH, other nonheterosexual; GSA, gay-straight alliance; PIQ, Personal Information Questionnaire (Pilkington \& D’Augelli, 1995); GAL-Q, Gay and Lesbian Questionnaire (Savin-Williams, 1989); RSES, Rosenberg Self-Esteem Scale (Rosenberg, 1965; 1979; 1989); CSES, Collective Self-Esteem Scale (Luhtanen \& Crocker, 1992); PWBS, Psychological Well-Being Scale (Ryff, 1989); LGBT-SES, LGBT Self-Esteem Scale (Shidlo, 1994); SDQ, Self-Description Questionnaire (Marsh \& O'Neill, 1982); SPPA, Self-Perception Profile for Adolescents (Harter, 1982); PSS-Fa, Perceived Social Support-Family (Procidano \& Heller, 1983); PSS-Fr, Perceived Social Support-Friends (Procidano \& Heller, 1983); SCS, Social Connectedness Scale (Lee \& Robbins, 1995); MOGS, Measure Of Gay-related Stressors (Lewis, 2001); CES-DS, Center for Epidemiology Studies-Depression Scale (Radloff, 1977); RCSS, Religious Comfort and Strain Scale (Yali \& Exline, 2004, as cited in Dahl \& Galliher, 2010); BMMRS, Brief Multidimensional Measure of Religiosity/Spirituality (Fetzer Institute, 1999); SNS, Support Network Survey (Berger, 1992); NRI, Network of Relations Inventory (Furman, 1996); ESM, Experience Sampling Method (Csikszentmihalyi \& Larson, 1987); BSI, Brief Symptom Inventory (Derogatis \& Spencer, 1982); FAS, Family Acceptance Scale (Ryan et al., 2009); GAD, Generalized Anxiety Disorder (Spitzer et al., 2006); LEC, Life Events Checklist (Johnson \& McCutcheon, 1980); DSM-III-R, Diagnostic and Statistical Manual of Mental Disorders-3rd edition-Revised (APA-American Psychiatric Association, 1987); PSS, Perceived Social Support (Procidano \& Heller, 1983); SOS, Social Obstruction Scale (Gurley, 1990, as cited in Rosario et al., 2011); MCSDS, Marlowe-Crowne Social Desirability Scale (Crowne \& Marlowe, 1964); LGBIS, Lesbian/Gay/Bisexual Identity Scale (Mohr \& Fassinger, 2000); MSPSS, Multidimensional Scale of Perceived Social Support (Zimet et al., 1988); GHQ, General Health Questionnaire (Goldberg, 1972); BHS, Beck Hopelessness Scale (Beck et al., 1974); BDI-II, Beck Depression Inventory (Beck, 1996); ISID, Index of Sexual Identity Disclosure (D'Augelli, 2002); NHAI, Nungesser Homosexual Attitudes Inventory (Nungesser, 1983); GRSE, Gay-Related Stressful Events (Rosario et al., 2002); ADM, Adolescent Disclosure Measure (Stattin \& Kerr, 2000); EARSI, Early Adolescent Role Strain Inventory (Fenzel, 1989a, 1989b, 2000); MBRS, Mentor Behavior Rating Scale (de Bruyn, 2004); ISQ, Identification with School Questionnaire (Voelkl, 1996a, 1996b); SSS, Social Support Scale (Vincke \& Bolton, 1996). 
The majority of studies were conducted in North America $(n=18)$, such as in the United States, Canada, or the two jointly. Only 2 out of the 20 studies originated from Europe, one from Belgium and the other from the Netherlands. The number of participants per study varied between 90 and 866, with a total number of 4865 participants across all studies. Of this number, data from 4755 were analyzed, which provides a satisfactory overall participation rate. However, the five studies where participants were omitted or participants withdrew had relatively low sample sizes.

Fourteen of the 20 studies employed a cross-sectional design, 9 of which recruited participants from all sexual minorities; 3 included lesbian, gay, and bisexual participants; and one included only lesbian and gay participants. The remaining six studies used a longitudinal design, three of which included participants of all sexual minorities; one included lesbian, gay, and bisexual participants; and one recruited solely lesbian and gay participants. All 20 studies used an individual assessment method; no group assessment had been conducted. Apart from one study, recruitment in all studies was conducted through either convenience sampling or snowball sampling; only one study (Blais et al., 2014) recruited participants from the general public. In the samples of 14 studies, males and females were equally represented; of the remaining 6 studies, 4 had an underrepresentation of female participants, 1 had an underrepresentation of male participants; and 1 utilized an exclusively male sample.

All 20 studies used adolescents and young adults as participants, and all reported participants' ages ranged between 13 and 25 across all the papers.

Four of the 20 studies used a mainly Caucasian sample. Seven out of 20 studies used an exclusively urban sample. Additionally, of the 20 studies included in this review, only 3 used solely gay and lesbian participants, whereas 17 included other sexual orientations as well.

\section{Measurement of sexual orientation}

All studies assessed participants' sexual orientation, but this assessment was done with a wide variety of methods. Only three studies used measurement scales to establish sexual orientation of participants. These scales were the Personal Information Questionnaire (Pilkington \& D’Augelli, 1995), the Gay and Lesbian Questionnaire (Savin-Williams, 1989), and the Kinsey Scale (Kinsey et al., 1948). The other 17 studies used mainly self-identification questionnaires, demographic and sociodemographic assessment, or questions on participants' background and sexual orientation history to establish their eligibility for participation, as well as using the sampling site itself, such as LGBT community centers, to find eligible participants. Sexual orientation in the included studies was assessed in a variety of ways with no overlap. However, all studies aimed to exclude exclusively heterosexual participants.

\section{Measurement of self-concept}

All studies assessed self-concept; however, only one domain of self-concept, as a key indicator, was used, which was self-esteem. With the exception of 4 studies, all studies used the full 10-item Rosenberg Self-Esteem Scale (RSES; Rosenberg, 1965, 1979, 1989 ) to assess self-esteem as a measurement of self-concept, and 1 study used 6 items of the RSES. Other measurements of self-concept included the Collective Self-Esteem Scale (CSES; Luhtanen \& Crocker, 1992), the LGBT Self-Esteem Scale (Shidlo, 1994), the Self-Description Questionnaire (Marsh \& O'Neill, 1982), and the Self-Perception Profile for Adolescents (Harter, 1982). It is apparent from the list outlined that most studies in this review showed congruency in the measurement used to assess self-concept, which provides a moderate to strong basis to compare study findings.

\section{Global self-concept measures}

As it was pointed out earlier, self-concept is a collection of several domains, of which the most studied in relation to gay and lesbian youth is the domain of self-esteem. None of the studies included in this review examined global self-concept.

\section{Global self-esteem measures}

Out of the 20 studies, 16 examined global self-esteem by administering the RSES (Rosenberg, 1965, 1979, 1989). One study (Detrie \& Lease, 2007) used the CSES (Luhtanen \& Crocker, 1992) to assess global self-esteem. Out of the 20 studies, 3 (Vincke \& van Heeringen, 2002; Blais et al, 2014; Wilkerson et al., 2017) did not assess global self-esteem.

\section{Measurement of social context}

The studies included in this review looked at various aspects of social context, using a variety of measurement scales. These measurements were Experience Sampling Method (Csikszentmihalyi \& Larson, 1987), Perceived Social SupportFamily and Friends (Procidano \& Heller, 1983), Social Connectedness Scale (Lee \& Robbins, 1995), Measure of GayRelated Stressors (Lewis, 2001), Support Network Survey (Berger, 1992), Center for Epidemiology Studies-Depression 
Scale (Radloff, 1977), Religious Comfort and Strain Scale (Dahl \& Galliher, 2010), Brief Multidimensional Measure of Religiosity/Spirituality (Fetzer Institute, 1999), Network of Relations Inventory (Furman, 1996), Brief Symptom Inventory (Derogatis \& Spencer, 1982), Family Acceptance Scale (Ryan et al., 2009), Generalized Anxiety Disorder (Spitzer et al., 2006), Life Events Checklist (Johnson \& McCutcheon, 1980), Diagnostic and Statistical Manual of Mental Disorders-3rd edition-Revised (APA-American Psychiatric Association, 1987), Social Obstruction Scale (Rosario et al., 2011), MarloweCrowne Social Desirability Scale (Crowne \& Marlowe, 1964), Lesbian/Gay/Bisexual Identity Scale (Mohr \& Fassinger, 2000), Multidimensional Scale of Perceived Social Support (Zimet et al., 1988), General Health Questionnaire (Goldberg, 1972), Beck Hopelessness Scale (Beck et al., 1974), Beck Depression Inventory (Beck, 1996), Index of Sexual Identity Disclosure (D’Augelli, 2002), Nungesser Homosexual Attitudes Inventory (Nungesser, 1983), Gay-Related Stressful Events (Rosario et al., 2002), Adolescent Disclosure Measure (Stattin \& Kerr, 2000), Early Adolescent Role Strain Inventory (Fenzel, 1989a, 1989b, 2000), Mentor Behavior Rating Scale (de Bruyn, 2004), Identification with School Questionnaire (Voelkl, 1996a, 1996b), and Social Support Scale (Vincke \& Bolton, 1996). The selection of the social context to be assessed in the individual studies was determined by the relevant research questions and by the nature of the sample used. The social context examined most often were family acceptance of sexual orientation, friends' attitudes, events related to victimization, participation in LGBT youth organizations, and mental health issues of the participants. It is apparent from the list outlined that the measurement of social context shows significant discrepancies, which sets obstacles to comparing the studies in relation to which areas of social context were measured and how.

\section{Summary of methodological characteristics}

In summary, the assessment of social context and self-concept was done using various measurement scales. Despite having used the RSES (Rosenberg, 1965, 1979, 1989) to examine selfconcept, all studies assessed social context with differing measurement scales. This produces methodological issues in the effort of comparing findings.

\section{Study findings}

Table 3 represents a summary of the main findings of the chosen studies.

Conclusions and findings inferred from the studies on the links between self-concept and social context will be examined here. It will be investigated whether the studies were able to establish a relationship between these two concepts. This will be performed in a qualitative manner and will not compare statistical findings.

\section{Relationship between self-concept and sexual identification}

Findings of studies on the relationship between self-concept and sexual identification were contradictory. One study found that self-esteem has a significant negative correlation with sexual orientation conflict (Dahl \& Galliher, 2010) and one study reported a positive correlation between self-esteem and sexual identification comfort (Hershberger \& D’Augelli, 1995). In contrast, Savin-Williams (1989) found no association between self-esteem and sexual orientation comfort. In addition, one study found that although concealing one's identity in school was negatively correlated with self-esteem, being out in school showed no significant correlation with self-esteem (Russel et al., 2014). One study found that same-sex attraction was negatively correlated with self-esteem (Bos et al., 2008). One study showed slightly higher self-esteem scores for lesbian youth than for gay youth (Savin-Williams, 1989). Contrastingly, two studies presented finding no significant differences between self-esteem and sexual orientation (Grossman \& Kerner, 1998; Bauermeister et al., 2010). Additionally, two studies found that self-esteem was positively correlated with LGBT-esteem and LGBQ (lesbian, gay, bisexual, questioning) identity salience (Snapp et al., 2015; Woodford et al., 2015). Out of 20 studies, 11 did not examine the relationship between self-concept and sexual identification measures.

\section{Relationship between social context and self-concept}

Out of 20 studies, 9 found a strong positive association between social context relating to family and friends-that displays as social support from family and friends, social acceptance, and social connectedness-and self-esteem (Savin-Williams, 1989; Snapp et al., 2015; Rosario et al., 2005; Wilkerson et al., 2017; Vincke \& van Heeringen, 2002; Bauermeister et al., 2010; Rosario et al., 2011; Bos et al., 2008). In contrast, whereas one study (Detrie \& Lease, 2007) found a strong positive correlation between social support from friends and self-esteem, they reported finding no correlation between social support from family and self-esteem. Two studies reported a positive association between social desirability and self-esteem (Rosario et al., 2005; Rosario et al., 2011). Another study revealed a positive correlation between family relations and self-esteem (Hershberger \& D’Augelli, 1995), but no significant correlation between family protection and self-esteem, or between family 
Table 3. Findings of studies

\begin{tabular}{|c|c|c|c|c|}
\hline$\#$ & Author (Year) & $\begin{array}{l}\text { Self-Concept vs. } \\
\text { Sexual Identification }\end{array}$ & Social Context vs. Self-Concept & Social Context vs. Sexual Identification \\
\hline 1 & $\begin{array}{l}\text { Detrie \& Lease } \\
\text { (2007) }\end{array}$ & & $\begin{array}{l}\text { Positive correlation between social } \\
\text { support from friends and self-esteem } \\
\text { ( } r=0.68, p<0.001 \text { ) } \\
\text { No correlation between social support } \\
\text { from family and self-esteem ( } r=0.01 \text { ) } \\
\text { Positive correlation between social } \\
\text { connectedness and self-esteem ( } r=0.30 \text {, } \\
p<0.001) \\
\text { Collective self-esteem positively related } \\
\text { to self-acceptance ( } r=0.38, p<0.001 \text { ) } \\
\text { Self-esteem positively related to social } \\
\text { support from family }(r=0.41, p<0.001) \\
\text { Self-esteem positively related to social } \\
\text { support from friends ( } r=0.41, p<0.001)\end{array}$ & \\
\hline 2 & $\begin{array}{l}\text { Dahl \& Galliher } \\
\text { (2010) }\end{array}$ & $\begin{array}{l}\text { Self-esteem negatively } \\
\text { correlated with sexual } \\
\text { orientation conflict } \\
(r=-0.399, p<0.01)\end{array}$ & $\begin{array}{l}\text { Self-esteem positively correlated with } \\
\text { positive God }(r=0.254, p<0.01) \text { and with } \\
\text { positive faith }(r=0.195, p<0.05) \\
\text { Self-esteem negatively correlated with } \\
\text { negative God }(r=-0.366, p<0.01) \text { and with } \\
\text { fear and guilt }(r=-0.404, p<0.01)\end{array}$ & $\begin{array}{l}\text { Positive correlation between sexual } \\
\text { orientation conflict, and negative God } \\
(r=0.240, p<0.01) \text { and fear and guilt } \\
(r=0.370, p<0.05)\end{array}$ \\
\hline 3 & $\begin{array}{l}\text { Grossman \& } \\
\text { Kerner (1998) }\end{array}$ & $\begin{array}{l}\text { No significant } \\
\text { differences between } \\
\text { self-esteem scores for } \\
\text { gay males }(M=19.7576, \\
n=58, S D=4.92) \text { and } \\
\text { lesbians }(M=19.00, n=32, \\
S D=5.83)\end{array}$ & $\begin{array}{l}\text { Higher self-esteem is a moderately strong } \\
\text { predictor of lower emotional distress in } \\
\text { gay males }\left(R=0.26, F_{(2,53)}=9.36, p<.001\right) \text { and } \\
\text { a strong predictor in lesbians }\left(R=0.51, F_{(2,}\right. \\
\left.{ }_{29}=14.85, p<0.001\right)\end{array}$ & $\begin{array}{l}\text { No significant differences in satisfaction } \\
\text { with support scores between gay males } \\
(M=3.69, n=58, S D=0.53) \text { and lesbians } \\
(M=3.66, n=32, S D=0.57) \\
\text { No significant differences in emotional } \\
\text { distress scores between gay males } \\
(M=1.17, n=58, S D=0.75) \text { and lesbians } \\
(M=1.23, n=32, S D=0.79)\end{array}$ \\
\hline 4 & $\begin{array}{l}\text { loverno et al. } \\
\text { (2016) }\end{array}$ & & $\begin{array}{l}\text { GSA presence leads to no changes in self- } \\
\text { esteem from } T_{1}(M=21.14, n=327, S D=5.64) \\
\text { to } T_{2}(M=20.72, n=327, S D=5.34)\end{array}$ & N/A \\
\hline 5 & $\begin{array}{l}\text { Poteat et al. } \\
\text { (2015) }\end{array}$ & $\mathrm{N} / \mathrm{A}$ & $\begin{array}{l}\text { Victimization negatively correlated with } \\
\text { self-esteem }(r=-0.18, p<0.5) \\
\text { Higher perception of support from GSA } \\
\text { predicted higher scores on self-esteem } \\
(B=0.12, p<0.05)\end{array}$ & $\mathrm{N} / \mathrm{A}$ \\
\hline 6 & $\begin{array}{l}\text { Hershberger \& } \\
\text { D’Augelli (1995) }\end{array}$ & $\begin{array}{l}\text { Self-esteem positively } \\
\text { correlated with sexual } \\
\text { identification comfort } \\
(r=0.33, p<0.05)\end{array}$ & $\begin{array}{l}\text { Self-esteem positively correlated with } \\
\text { family relations ( } r=0.11, p<0.05) \\
\text { Self-esteem showed no significant } \\
\text { correlation with family acceptance } \\
(r=0.03) \text {, or with family protection ( } r=-0.01) \\
\text { Self-esteem negatively correlated with } \\
\text { mental health problems }(r=-0.46, p<0.05)\end{array}$ & $\begin{array}{l}\text { Sexual identification comfort positively } \\
\text { correlated with family acceptance } \\
(r=0.18, p<0.05) \text {, with family protection } \\
(r=0.21, p<0.05) \text {, and with family relations } \\
(r=0.44, p<0.05)\end{array}$ \\
\hline 7 & $\begin{array}{l}\text { Savin-Williams } \\
\text { (1989) }\end{array}$ & $\begin{array}{l}\text { Self-esteem scores } \\
\text { slightly higher for } \\
\text { lesbians ( } M=22.18 \text { ) than } \\
\text { for gay males ( } M=21.98 \text { ) } \\
\text { Self-esteem shows no } \\
\text { association with sexual } \\
\text { orientation comfort }\end{array}$ & $\begin{array}{l}\text { Self-esteem shows no association with } \\
\text { parental acceptance, contact with parents, } \\
\text { relationship with father, or marital status } \\
\text { of parents } \\
\text { Lesbians' self-esteem shows significant } \\
\text { correlation with mother's age ( } r=-0.23, \\
p<0.02 \text { ) and satisfaction with relationship } \\
\text { with mother ( } r=-0.26, p<0.01) \\
\text { Gay males' self-esteem shows significant } \\
\text { correlation with being out to their mother } \\
(r=-0.15, p<0.03) \text { and with satisfaction with } \\
\text { mother }(r=-0.22, p<0.003) \text { and with father } \\
(r=-0.22, p<0.002)\end{array}$ & $\begin{array}{l}\text { Lesbians scored higher ( } M=2.71) \text { than } \\
\text { gay males ( } M=2.41) \text { on being out to their } \\
\text { father } \\
\text { Lesbians and gay males scored similar } \\
\text { ( } M=2.14 \text { and } M=2.15 \text {, respectively) on } \\
\text { being out to their mother }\end{array}$ \\
\hline
\end{tabular}


Table 3. Findings of studies

\begin{tabular}{|c|c|c|c|c|}
\hline$\#$ & Author (Year) & $\begin{array}{l}\text { Self-Concept vs. } \\
\text { Sexual Identification }\end{array}$ & Social Context vs. Self-Concept & Social Context vs. Sexual Identification \\
\hline 8 & $\begin{array}{l}\text { Snapp et al. } \\
\text { (2015) }\end{array}$ & $\begin{array}{l}\text { Self-esteem positively } \\
\text { correlated with LGBT- } \\
\text { esteem }(r=0.41, p<0.05)\end{array}$ & $\begin{array}{l}\text { Self-esteem positively correlated with } \\
\text { support from friends about being LGBT } \\
\text { ( } r=0.19, p<0.05) \\
\text { Self-esteem positively correlated with the } \\
\text { availability of LGBT books and magazines } \\
\text { ( } r=0.16, p<0.05) \\
\text { Family acceptance predicted self-esteem } \\
(B=0.38, p<0.001) \\
\text { Support from friends about being LGBT } \\
\text { predicted self-esteem }(B=0.15, p<0.05)\end{array}$ & $\begin{array}{l}\text { Being out to friends positively correlated } \\
\text { with LGBT-esteem }(r=0.20, p<0.05) \\
\text { Support from friends about being LGBT } \\
\text { positively correlated with LGBT-esteem } \\
\text { ( } r=0.19, p<0.05 \text { ) } \\
\text { Being out to family, friends, and others } \\
\text { predicted LGBT-esteem ( } B=0.31, p<0.001 \text { ) } \\
\text { Family Acceptance predicted LGBT- } \\
\text { Esteem ( } B=.36, p<.001) \text {. }\end{array}$ \\
\hline 9 & $\begin{array}{l}\text { Woodford et al. } \\
\text { (2015) }\end{array}$ & $\begin{array}{l}\text { LGBQ identity salience } \\
\text { positively correlated } \\
\text { with self-esteem } \\
(r=0.17, p<0.05)\end{array}$ & $\begin{array}{l}\text { Self-esteem negatively correlated with } \\
\text { anxiety ( } r=-0.55, p \leqslant 0.001) \\
\text { Self-esteem negatively correlated with } \\
\text { perceived stress ( } r=-0.71, p \leqslant 0.001 \text { ) }\end{array}$ & $\begin{array}{l}\text { Relationship recognition is negatively } \\
\text { correlated with } L G B Q \text { identity salience } \\
\text { ( } r=-0.15, p<0.05) \\
\text { Distal environmental microaggressions } \\
\text { were positively correlated with LGBQ } \\
\text { identity salience ( } r=0.30, p \leqslant 0.001)\end{array}$ \\
\hline 10 & $\begin{array}{l}\text { Rotheram- } \\
\text { Borus et al. } \\
\text { (1995) }\end{array}$ & N/A & $\begin{array}{l}\text { Self-esteem positively but not significantly } \\
\text { correlated with gay-related stressors } \\
(r=0.15, p<0.05) \text {, with academic stressors } \\
(r=0.03, p<0.05) \text {, with other life stressors } \\
(r=0.15, p<0.05) \text {, or with emotional distress } \\
(r=0.11, p<0.05)\end{array}$ & N/A \\
\hline 11 & $\begin{array}{l}\text { D’Augelli \& } \\
\text { Hershberger } \\
\text { (1993) }\end{array}$ & N/A & $\begin{array}{l}\text { Self-esteem negatively correlated with } \\
\text { mental health issues }(r=-0.44, p<01) \\
\text { Self-esteem negatively correlated with } \\
\text { depression }(r=-0.49, p<0.01) \\
\text { Self-esteem negatively correlated with } \\
\text { interpersonal sensitivity }(r=-0.41, p<0.01)\end{array}$ & $\mathrm{N} / \mathrm{A}$ \\
\hline 12 & $\begin{array}{l}\text { Russel et al. } \\
\text { (2014) }\end{array}$ & $\begin{array}{l}\text { Self-esteem negatively } \\
\text { correlated with hiding } \\
\text { at school ( } r=-0.21, \\
p<0.001) \\
\text { Self-esteem showed no } \\
\text { significant correlation } \\
\text { with being out in school } \\
(r=0.10)\end{array}$ & $\begin{array}{l}\text { Self-esteem negatively correlated with } \\
\text { LGBT victimization ( } r=-0.24, p<0.05 \text { ) } \\
\text { Self-esteem negatively correlated with } \\
\text { depression ( } r=-0.47, p<0.001 \text { ) } \\
\text { Self-esteem positively correlated with life } \\
\text { satisfaction ( } r=0.34, p<0.001 \text { ) }\end{array}$ & $\begin{array}{l}\text { Hiding at school was positively } \\
\text { correlated with LGBT victimization } \\
(r=0.14, p<0.05) \\
\text { Hiding at school positively correlated } \\
\text { with depression ( } r=0.24, p<0.001) \\
\text { Hiding at school negatively correlated } \\
\text { with life satisfaction ( } r=-0.14, p<0.05) \\
\text { Being out at school positively correlated } \\
\text { with LGBT victimization ( } r=0.29, p<0.001) \\
\text { Being out at school negatively correlated } \\
\text { with depression ( } r=-0.22, p<0.001) \\
\text { Being out at school positively correlated } \\
\text { with life satisfaction }(r=0.16, p<0.05)\end{array}$ \\
\hline
\end{tabular}




\begin{tabular}{|c|c|c|c|c|}
\hline$\#$ & Author (Year) & $\begin{array}{l}\text { Self-Concept vs. } \\
\text { Sexual Identification }\end{array}$ & Social Context vs. Self-Concept & Social Context vs. Sexual Identification \\
\hline 13 & $\begin{array}{l}\text { Rosario et al. } \\
(2005)\end{array}$ & N/A & $\begin{array}{l}\text { At baseline: } \\
\text { Self-esteem negatively correlated with } \\
\text { depression }(r=-0.62, p<0.05) \text {, with anxiety } \\
\text { ( } r=-0.35, p<0.05), \text { and with conduct } \\
\text { problems ( } r=-0.16, p<0.05) \\
\text { Self-esteem positively correlated with } \\
\text { social support from family }(r=0.27, p<0.05) \\
\text { and friends ( } r=0.28, p<0.05), \text { and with } \\
\text { social desirability }(r=0.25, p<0.05) \\
\text { Self-esteem negatively correlated with } \\
\text { negative social relationships ( } r=-0.44, \\
p<0.05) \\
\text { At } 6-\text { month assessment: } \\
\text { Self-esteem negatively correlated with } \\
\text { depression ( } r=-0.54, p<0.05) \text { and with } \\
\text { anxiety ( } r=-0.28, p<0.05) \text {. Correlation } \\
\text { between self-esteem and conduct } \\
\text { problems was negative but not significant } \\
(r=-0.10) \\
\text { At } 12-\text { month assessment: } \\
\text { Self-esteem negatively correlated with } \\
\text { depression ( } r=-0.37, p<0.05) \text { and with } \\
\text { anxiety ( } r=-0.23, p<0.05) \text {. Correlation } \\
\text { between self-esteem and conduct } \\
\text { problems was negative but not significant } \\
(r=-0.02)\end{array}$ & N/A \\
\hline 14 & $\begin{array}{l}\text { Blais et al. } \\
\text { (2014) }\end{array}$ & $\mathrm{N} / \mathrm{A}$ & $\begin{array}{l}\text { Self-esteem negatively correlated with } \\
\text { homophobic bullying }(r=-0.171, p<0.05) \\
\text { Self-esteem negatively correlated with } \\
\text { internalized homophobia ( } r=-0.171, p<0.05) \\
\text { Internalized homophobia positively } \\
\text { correlated with homophobic bullying } \\
(r=0.388, p<0.05) \\
\text { Relationship between self-esteem } \\
\text { and homophobic bullying mediated by } \\
\text { internalized homophobia }\end{array}$ & N/A \\
\hline 15 & $\begin{array}{l}\text { Wilkerson et al. } \\
\text { (2017) }\end{array}$ & $\mathrm{N} / \mathrm{A}$ & $\begin{array}{l}\text { Increased social support associated with } \\
\text { increased self-esteem }(B=-0.72, p<0.05) \\
\text { Hatch Youth attendance was associated } \\
\text { with increasing self-esteem }\left(T_{1}: B=0.08 \text {, }\right. \\
\left.p<0.05 ; T_{2}: B=0.30, p<0.05\right)\end{array}$ & N/A \\
\hline 16 & $\begin{array}{l}\text { Vincke \& van } \\
\text { Heeringen } \\
\text { (2002) }\end{array}$ & $\mathrm{N} / \mathrm{A}$ & $\begin{array}{l}\text { Self-esteem positively correlated with } \\
\text { parents' awareness of sexual orientation } \\
\text { at } T_{1} \text { and } T_{2} \text {, but these correlations } \\
\text { are not significant ( } r=0.09 \text { and } r=0.09, \\
\text { respectively) } \\
\text { Self-esteem negatively correlated with } \\
\text { parent acceptance at } T_{1}(r=-0.03) \text { and } \\
\text { positively correlated at } T_{2}(r=0.08) \text {, but } \\
\text { these correlations are not significant } \\
\text { Self-esteem positively correlated with } \\
\text { satisfaction with lesbian/gay friendship } \\
\text { relations at } T_{1}(r=0.20, p<0.05) \text { and at } T_{2} \\
\text { (r=0.26, } p<0.05) \\
\text { Self-esteem positively correlated with } \\
\text { confidant support at } T_{1}(r=0.41, p<0.05) \text { and } \\
\text { at } T_{2}(r=0.41, p<0.05)\end{array}$ & N/A \\
\hline
\end{tabular}


Table 3. Findings of studies

\begin{tabular}{|c|c|c|c|c|}
\hline$\#$ & Author (Year) & $\begin{array}{l}\text { Self-Concept vs. } \\
\text { Sexual Identification }\end{array}$ & Social Context vs. Self-Concept & Social Context vs. Sexual Identification \\
\hline 17 & $\begin{array}{l}\text { Bauermeister } \\
\text { et al. }(2010)\end{array}$ & $\begin{array}{l}\text { Self-esteem was not } \\
\text { associated with Kinsey } \\
\text { Scale scores }\end{array}$ & $\begin{array}{l}\text { Males: } \\
\text { Self-esteem at } T_{2} \text { positively associated } \\
\text { with self-esteem at } T_{1}(B=0.47, p \leqslant 0.001) \text {, } \\
\text { with number of friends aware of sexual } \\
\text { identity at } T_{2}(B=0.15, p \leqslant 0.05) \text {, and with } \\
\text { social support at } T_{2}(B=0.17, p \leqslant 0.05) \\
\text { Females: } \\
\text { Self-esteem at } T_{2} \text { positively associated } \\
\text { with self-esteem at } T_{1}(B=0.63, p \leqslant 0.001) \\
\text { and with social support } T_{2}(B=0.20, p \leqslant 0.01)\end{array}$ & N/A \\
\hline 18 & $\begin{array}{l}\text { Rosario et al. } \\
\text { (2011) }\end{array}$ & & $\begin{array}{l}\text { Self-esteem positively associated with } \\
\text { social desirability ( } r=0.28, p<0.05 \text { ) } \\
\text { Self-esteem was positively associated } \\
\text { with family support ( } r=0.26, p<0.05 \text { ) } \\
\text { Self-esteem negatively associated with } \\
\text { negative social relationships ( } r=-0.36, \\
p<0.05 \text { ) } \\
\text { Highly integrated youth reported higher } \\
\text { self-esteem }\end{array}$ & $\begin{array}{l}\text { Highly integrated youth reported less } \\
\text { anxiety and depression, and fewer } \\
\text { conduct problems }\end{array}$ \\
\hline 19 & $\begin{array}{l}\text { Bos et al. } \\
\text { (2008) }\end{array}$ & $\begin{array}{l}\text { Sexual attraction } \\
\text { negatively correlated } \\
\text { with self-esteem } \\
(r=-0.14, p<0.001)\end{array}$ & $\begin{array}{l}\text { Self-esteem positively correlated with } \\
\text { disclosure to father }(r=0.26, p<0.001) \text {, with } \\
\text { disclosure to mother }(r=0.18, p<0.001) \text {, } \\
\text { with social acceptance }(r=0.38, p<0.001) \text {, } \\
\text { and with respect from mentor }(r=0.15 \text {, } \\
p<0.001) \\
\text { Self-esteem negatively correlated with } \\
\text { peer role strain }(r=-0.21, p<0.001) \text {, and } \\
\text { with depression ( } r=-0.66, p<0.001)\end{array}$ & $\begin{array}{l}\text { Sexual attraction negatively correlated } \\
\text { with disclosure to father }(r=-0.10, \\
p<0.01) \text {, with social acceptance }(r=-0.09 \text {, } \\
p<0.01) \text {, with respect from mentor } \\
(r=-0.09, p<0.01) \text {, and with school } \\
\text { identification }(r=-0.09, p<0.01) \\
\text { Sexual attraction negatively correlated } \\
\text { with disclosure to mother, but } \\
\text { correlation was not significant }(r=-0.04) \\
\text { Sexual attraction positively correlated } \\
\text { with peer role strain }(r=0.10, p<0.01) \text { and } \\
\text { with depression }(r=0.26, p<0.001)\end{array}$ \\
\hline 20 & $\begin{array}{l}\text { Toomey et al. } \\
\text { (2011) }\end{array}$ & & $\begin{array}{l}\text { Self-esteem positively associated with } \\
\text { GSA presence }(r=0.12, p<0.01) \\
\text { Self-esteem showed no significant } \\
\text { association with GSA participation }(r=0.06) \\
\text { or with perceived GSA effectiveness } \\
(r=0.05)\end{array}$ & \\
\hline
\end{tabular}

GSA, gay straight alliance.

acceptance and self-esteem. Furthermore, two studies (Rosario et al., 2005; Rosario et al., 2011) found a negative correlation between negative social relationships and self-esteem.

In relation to the religious context, one study reported a strong positive association between the experience of positive faith and positive God experience, and self-esteem (Dahl \& Galliher, 2010). This study also found a negative association between fear and guilt of religious consequences and a negative God experience, and self-esteem. Additionally, their findings indicated that negative religious experiences have a deeper impact on the mental health of sexual minorities than do positive religious experiences.
With regard to the social setting within the community, two studies (Snapp et al., 2015; Wilkerson et al., 2017) found that availability of LGBT resources-for instance, books, magazines and youth groups (such as Hatch Youth) —-was positively correlated with self-esteem. One study (Rotheram-Borus et al., 1995) presented a negative but not significant correlation between other life stressors (including but not exclusive to the domains of family, self, peers, health, and moving from place to place) and self-esteem.

Additionally, regarding school context, two studies (Toomey et al., 2011; Poteat et al., 2015) found that GSA presence and being out to peers was associated with higher self-esteem. Contrastingly, one study (Ioverno et al., 2016) showed that 
GSA presence led to no changes in self-esteem scores over time. Similarly, another study (Toomey et al., 2011) reported no significant association between self-esteem, and GSA participation or perceived GSA effectiveness. Three studies (Rotheram-Borus et al., 1995; Blais et al., 2014; Poteat et al., 2015) found that stressors in the school environment-such as gay-related stressors, academic stressors, victimization and homophobic bullying-were negatively correlated with selfesteem, as well as with internalized homophobia. Moreover, one study (Bos et al., 2008) showed a negative association between peer role strain and self-esteem.

Furthermore, five studies (D’Augelli \& Hershberger, 1993; Hershberger \& D’Augelli, 1995; Rosario et al., 2005; Bos et al., 2008; Woodford et al., 2015) found significant negative correlations between mental health issues-manifesting in forms of depression, anxiety, conduct problems, interpersonal sensitivity, perceived stress, and general mental health-and self-esteem. Although one study (Rosario et al., 2005) found longitudinal associations between depression and anxiety, and self-esteem, they revealed no statistically significant correlation between conduct problems and self-esteem over time.

\section{Relationship between social context and sexual identification}

Eleven out of 20 studies did not assess the relationship between social context and sexual identification measures. All nine studies that did examine this relationship agree that a negative social context would presumably promote problems with one's sexual identification.

From a religious perspective, one study found a positive correlation between sexual orientation conflict and a negative God experience, as well as between sexual orientation conflict and fear and guilt (Dahl \& Galliher, 2010).

One study (Hershberger \& D’Augelli, 1995) reported that sexual identification comfort was positively correlated with family acceptance, family protection, and family relations. Similarly, being out at school positively correlated with life satisfaction, and negatively correlated with depression (Russel et al., 2014). The same study also found that hiding one's sexual orientation at school was positively correlated with depression and negatively correlated with life satisfaction. Interestingly, the study also reported that both hiding one's sexuality in school and being out were positively correlated with LGBT victimization. Comparable findings were demonstrated by another study (Snapp et al., 2015), suggesting that being out and identifying as having same-sex attraction, rather than concealing one's sexual identity, was positively correlated with LGBT-esteem.
Although one study reported that specific sexual orientation meant no differences in scores for satisfaction with support or for emotional distress (Grossman \& Kerner, 1998), another study (Bos et al., 2008) found that same-sex attraction was indeed positively correlated with peer role strain and depression. They also found that same-sex attraction showed a negative correlation with social acceptance, school identification, and disclosure of one's sexual identification to parents, all of which may lead to heightened levels of emotional distress.

\section{DISCUSSION}

The present paper provides a systematic review of the currently existing literature assessing the impact social context may have on the self-concept of gay and lesbian youth.

The aim was to investigate the links between social context and self-concept in gay and lesbian youth and to examine potential methodological threats in the literature. It was predicted that the reviewed literature would present methodological inconsistencies. As anticipated, results of the quality assessment implied reporting heterogeneity, in both study quality and reporting quality, within the literature on the self-concept of gay and lesbian youth. In relation to research, synthesis of study findings revealed that participant sampling and study design can often fundamentally influence the type of insight one might gain into the processes and mechanisms underlying the development of self-concept in gay and lesbian youth under the influence the social context they function in. As far as research is concerned, comparison of study findings demonstrated that most researchers appeared to have disregarded the various subgroups within a nonheterosexual orientation, and attempted to draw conclusions regarding sexual minority youth as a homogeneous group, still only focusing on self-esteem as a single domain within self-concept.

It was also predicted that a more positive social context would be associated with a more positive self-concept. The review revealed that previous studies investigated four main types of social context: school, friends and family, community, and religion, all of which were examined separately. With reference to self-concept, the studies included in the review mostly focused on one individual domain, instead of assessing selfconcept as a global dimension. Most of the papers examined self-esteem; evidence from findings indicates that it may be lower in gay and lesbian youth than in their heterosexual peers (Garofalo et al., 1998); however, it can also reach considerably higher levels when an optimal and positive social context is available (Detrie \& Lease, 2007; Rosario et al., 2011; Snapp et al., 2015; Wilkerson et al., 2017). Contrastingly, self-esteem might also significantly decrease through youth's interaction with 
a negative social environment where negative stereotyping is associated with being a gay male or a lesbian (D’Augelli \& Hershberger, 1993; Vincke \& van Heeringen, 2002; Bos et al., 2008; Woodford et al., 2015). These interactions, which are often infused with negative attributions and attitudes by others, can lead to sexual identity confusion and low selfesteem, essentially hindering the development of a healthy and positive self-concept (Garofalo et al., 1998). Hence, the findings of the present review support Higgins' (1987) self-discrepancy theory, underpinning the notion that by reaching a balance between their sexual identities and social expectations, gay and lesbian youth may achieve an optimal position that allows for a positive self-concept development by diminishing fear, shame, guilt, and rejection resulting from belonging to a sexual minority group.

Another aim of the current review was to examine whether highlighting the importance of social context would benefit intervention and support services, and families raising gay or lesbian youth. Comparing findings from the studies in relation to social context (either school, family and friends, community, or religion) uncovers that the more understanding and accepting gay and lesbian youth's environment is, the more likely it is that youth will develop a healthy self-conceptthat fully integrates their differing sexual orientation and identity-through an easier path (Hershberger \& D'Augelli, 1995; Bauermeister et al., 2010; Snapp et al., 2015). Nevertheless, it is necessary to acknowledge that although attitudes of the immediate social environment should be positive in order to aid self-concept development, not all members of the social context will have positive attributions toward alternative sexual orientations. As a result, communications and mediations regarding full integration of gay and lesbian youth need to be managed with realistic expectations in mind.

\section{Limitations of the present review}

Review and assessment of the included studies found several problems when progressing onto integrating findings from existing research. These mostly involved methodological issues and disparities.

Although overall attrition rates in the studies were not very high, looking at the detailed list it is observable that the highest rates occurred in the studies applying a longitudinal design. This essentially defies the purpose of a longitudinal design, and possibly leads to distorted results. In addition, most samples used were recruited from convenience populations, such as geographically limited, mostly urban high school and university areas, associations for nonheterosexual youth, as well as through snowball sampling. These population targets represent an issue with external validity because of the nonrepresentative nature of the samples, leading to unattainability in extrapolating study findings to the general population of nonheterosexual youth.

As pointed out earlier in the present review, social context is multifaceted, involving positive or negative attributions of others, social resources, and actual and perceived social support and attitudes from sources such as family, friends, school, community, or religious settings. Attitudes and available resources can fundamentally shape selfconcept. Nevertheless, not all individuals experience the same environment the same way. Hence, mediating and moderating variables may and should be considered when examining self-concept in a social context in relation to gay and lesbian youth.

The mechanism that makes positive social contacts useful is often not clear. Also, the definition and measurement of social support and perceived social support varies across studies. The lack of a solid definition makes synthesizing findings of the studies difficult and the relationship of social support with self-concept inconclusive.

It was pinpointed earlier that the definition of selfconcept occurred inconsistently throughout the literature. Additionally, the measurement of both self-concept and social context showed discrepancies across the studies included in this review. Although the measurement scales used to assess self-concept overlapped in the studies, most still only examined a single domain of self-concept. With the exception of two studies (Bos et al., 2008; Blais et al., 2014) that examined self-perception in addition to self-esteemall papers focused on only one domain of self-concept. Moreover, the measurement scales used to examine social context and sexual orientation were different in almost all the studies. Regarding social context, only one study (Dahl \& Galliher, 2010) explored the impact of a religious context; all other studies assessed the significance of the close social environment, such as family, friends, and peers, and that of the wider social context, such as school and community. This incongruence and lack of agreement on the choice of assessment instruments hinders making conclusive, direct comparisons between studies.

Whereas the problem with coherence of measurement of social context and self-concept is evident from the results of this review, this issue also occurs in relation to sexual identification. The concept of sexual minority lacks an 
adequate definition, which can be noted among the reviewed studies. Many of the papers discussed sexual orientation as a term interchangeable with the term "homosexual," without accounting for specific subgroups and their possible variance in their relations to social context and self-concept.

In addition to issues with comparing study findings, the present review is also limited by using only one rater to assess the quality of papers. Bias could be decreased and interrater reliability could be established by employing a second rater in assessing methodological quality of the included studies.

Focusing only on gay and lesbian youth may have also limited the findings of this review.

\section{Implications for research}

Future research could possibly explore a wider range of samples, such as with equal representation of urban and rural youth from a representative sample of the general public, as well as by conducting large-scale, international, longitudinal studies, preferably with the inclusion of heterosexual control samples. Also, combining findings from cross-cultural samples would enrich the insight into the link between social context and self-concept in relation to gay and lesbian youth and could also improve the external validity and generalizability of research findings. In addition, assessing all domains of selfconcept, rather than self-esteem only, might be beneficial in gaining a more cohesive view of its development path in gay and lesbian youth.

Moreover, including variables such as socioeconomic status, ethnicity, and religious affiliation would allow a more complex assessment of the mediating and moderating effect of social context variables. These attempts would enable a robust basis for making reliable comparisons between studies and allow for effectively synthesizing results. It would also permit for a more thorough analysis of findings, for drawing solid conclusions about the relationships between social context and self-concept, and for assessing their relations in a comparison between heterosexual and nonheterosexual youth.

The present review suggests that future research could aim to create and validate a composite measure for the three concepts separately (sexual identification, social context, self-concept), for which currently available validated measurement scales would serve as a basis. This would also further the possibility of making adequate comparisons between research studies in the examined field of literature.
Also, when examining the impact of social context on the self-concept of gay and lesbian youth, there is a strong need for extensive longitudinal research in order to establish the relationships between concepts over time and to identify the underlying mechanisms through which the interaction of these concepts influences the development of a healthy and positive self-concept.

In addition, future research could examine the relationships between social context and self-concept in gay and lesbian youth with the use of qualitative study designs. This method would allow a richer understanding of youth's subjective experiences. Similarly, qualitative studies could further explore the subjective experiences of those living in gay and lesbian youth's social environment, such as family, friends, peers, or teachers. Such studies might grant insight into findings that could serve as a basis for more solid and comprehensive intervention programs to aid the healthy self-concept development in gay and lesbian youth and to facilitate the evolution of a more tolerant and inclusive social structure.

\section{Implications for practice}

Review of the literature indicates that when the social context is positive and nurturing, there is a higher possibility for a healthy self-concept development in gay and lesbian youth. This is in line with the proposed idea of social context theory (Earle \& Earle, 1999) and suggests that social context has a vast impact and must be examined in relation to gay and lesbian youth's self-concept.

It is a clear finding of this review that the close social environment, such as family and friends are also affected by youth's sexual identification and, depending on their attitudes and perceptions, can have a positive buffering effect on youth's resilience resources, or can act as stressors and pose a negative impact. Hence, unhindered and fruitful communication between youth, family, and friends is essential in creating an easier path for gay and lesbian youth to develop a healthy self-concept.

Findings of this review on the negative relationship between mental health issues and self-concept clearly point to the need for effective psychological interventions for gay and lesbian youth. Decreasing the extent of negative social relationships is paramount in reducing emotional distress, and interventions aiming to help gay and lesbian youth must identify these relationships in order to be able to tailor their services to individual needs. Gay and lesbian youth are often treated as a homogeneous group with regard to their issues, and when it comes to their sexual identification, and when the concern 
is the revealing or concealing of their sexual orientations to their social environment.

It is be suggested that therapy session may be of use, including both youth and their parents, if the source of negativity is the parents, with a goal to resolve differences and achieve mutual understanding of emotions, needs, and expectations. It is also important that alongside identifying positive resources for youth to lean on, they also learn positive coping mechanisms to deal with difficult situations, with the loss of friends as a result of coming out, and to handle other negative peer feedbacks. Moreover, if the negative social relationships stem more from peers, it is suggested that gay and lesbian youth seek complementary peer relationships in the form of participation in gay- and lesbian-focused organizations. Higher self-esteem, which may hopefully be gained through these positive sources, may assist youth in their coming out process and consequently in becoming more integrated with their sexual identities.

Additionally, the review of literature indicates that whereas GSA presence in schools may have a slight positive impact on gay and lesbian youth's self-esteem, its presence might not, in itself, be enough of a positive social context measure to sufficiently support healthy self-concept development. It may be necessary for schools to implement specific policies and procedures (such as teacher training or curriculum inclusive of sexual minority issues) in relation to sexual orientation of students who are not exclusively heterosexual. Therefore, schools' support to GSA is fundamental as a starting point for creating a safer and more inclusive environment and for providing further education about LGBTQ (lesbian, gay, bisexual, transgender, questioning) issues to heterosexual peers.

As the findings of this review suggest that in most cases increased social support is associated with enhanced levels of self-esteem, which facilitates the development of a balanced self-concept, school psychologists and counselors, as well as GSAs, may plan to work together in working with sexual minority youth to increase their self-esteem as part of the process of achieving a positive and balanced self-concept. Moving outside of youth's personal social environment and examining the elements of the wider social context (heterosexism, noninclusive social policies, covert aggression through media, and negative community attitudes toward sexual minorities) is necessary in creating exhaustive policies, practices, and interventions. Also, intervention should include long-term follow-up, as homophobic bullying and victimization can have long-lasting negative effects, not only on self-concept, but generally on mental health and physical and emotional well-being of gay and lesbian youth.

In conclusion, the present review suggests that social context has a substantial impact on the self-esteem and on the selfperceptions of gay and lesbian youth. It also seems to influence the way they perceive others, and others' attributions and attitudes toward themselves. Even though the inconsistency of the measurement scales and other assessment tools used across the existing literature makes it problematic to produce a cohesive synthesis of study findings, it is evident from each individual study that social context in some way has a significant impact on self-concept. This provides important implications about the need for collaboration between gay and lesbian youth, their families and friends, schools, GSAs, community contexts, policy makers, and service providers, in designing and implementing positive social resources with the aim to facilitate healthy self-concept development in gay and lesbian youth. This cooperation is crucial, because addressing only the proximal social context may not yield thorough and satisfactory results.

\section{ACKNOWLEDGEMENTS}

No acknowledgements.

\section{ETHICAL APPROVAL}

As no new data were collected for this study (systematic review), no ethical approval was necessary.

\section{INFORMED CONSENT}

As no new data were collected for this study (systematic review), no informed consent was required.

\section{CONFLICTS OF INTEREST}

The authors declare no conflict of interest in conducting this review.

\section{FUNDING}

This review did not receive any specific grant from funding agencies in the public, commercial, or not-for-profit sectors. 


\section{REFERENCES}

American Psychiatric Association (1987). Diagnostic and Statistical Manual of Mental Disorders: DSM-III-R (3rd edn., Rev. edn.). American Psychiatric Association, Washington, DC.

Bagley, C., \& Tremblay, P. (1997). Suicidal Behaviors in Homosexual and Bisexual Males. Crisis: The Journal of Crisis Intervention and Suicide Prevention, 18(1), 24-34.

*Bauermeister, J., Johns, A., Sandfort, M., Eisenberg, M., Grossman, T., \& D’Augelli, G. (2010). Relationship Trajectories and Psychological Well-Being Among Sexual Minority Youth. Journal of Youth and Adolescence, 39(10), 1148-1163.

Beck, A. T. (1996). Beck Depression Inventory II. San Antonio, TX: Psychological Corporation.

Beck, A. T., Weisman, A., \& Lester, D. (1974). The measurement of pessimism: the hopelessness scale. Journal of Consulting and Clinical Psychology, 42, 861-865.

Bentall, Corcoran, Howard, Blackwood, \& Kinderman. (2001). Persecutory delusions: A review and theoretical integration. Clinical Psychology Review, 21(8), 1143-1192.

Berger, R. (1992). Passing and Social Support Among Gay Men. Journal of Homosexuality, 23(3), 85-98.

Berkman, L. (1995). Role of Social-Relations in Health Promotion. Psychosomatic Medicine, 57(3), 245-254.

*Blais, Martin, Gervais, Jesse, \& Hebert, Martine. (2014). Internalized homophobia as a partial mediator between homophobic bullying and self-esteem among youths of sexual minorities in Quebec (Canada)/Homofobia internalizada como mediador parcial do bullying homofobico e autoestima entre jovens de minorias sexuais em Quebec (Canada).(articulo en ingles). Ciencia \& Saude Coletiva, 19(3), 727-735.

*Bos, Henny M. W., Sandfort, Theo G. M., De Bruyn, Eddy H., \& Hakvoort, Esther M. (2008). Same-Sex Attraction, Social Relationships, Psychosocial Functioning, and School Performance in Early Adolescence. Developmental Psychology, 44(1), 59-68.

British Medical Journal. (2009). Preferred reporting items for systematic reviews and meta-analyses: the PRISMA statement: http://dx.doi.org/10.1136/bmj.b2535
California Healthy Kids Survey (2010). California Healthy Kids Survey: Survey Content \& Download. http://chks.wested.org/ administer/download

Campbell, J. (1990). Self-esteem and clarity of the self-concept. Journal of Personality and Social Psychology, 59(Sep 90), 538-549.

Clingman, J., \& Fowler, M. G. (1976). Gender Roles and Human Sexuality. Journal of Personality Assessment, 40(3), 276-284.

Crowne, D. P., \& Marlowe, D. (1964). The approval motive: Studies in evaluative dependence. Westport, CT: Greenwood.

Csikszentmihalyi M, Larson R (1987). Validity and Reliability of the Experience-Sampling Method. Journal of Nervous and Mental Disease, 175, 526-536.

*Dahl, A., \& Galliher, R. (2010). Sexual Minority Young Adult Religiosity, Sexual Orientation Conflict, Self-Esteem and Depressive Symptoms. Journal of Gay \& Lesbian Mental Health, 14(4), 271-290.

D’Augelli, A. R. (2002). Mental Health Problems among Lesbian, Gay, and Bisexual Youths Ages 14-21. Clinical Child Psychology and Psychiatry, 7(3), 439-462.

*D’Augelli, A., \& Hershberger, S. (1993). Lesbian, gay, and bisexual youth in community settings: Personal challenges and mental health problems. American Journal of Community Psychology, 21(4), 421-448.

de Bruyn, E. H. (2004). Development of the mentor behavior rating scale. School Psychology International, 25, 185-192.

Derogatis, L. R. (1993). BSI: Brief Symptom Inventory: Administration, scoring, and procedures manual. Minneapolis: National Computer Systems.

*Detrie, P., \& Lease, S. (2007). The Relation of Social Support, Connectedness, and Collective Self-Esteem to the Psychological Well-Being of Lesbian, Gay, and Bisexual Youth. Journal of Homosexuality, 53(4), 173-199.

Diamond, L.M., \& Lucas, S. (2004). SexualuMinority and Heterosexual Youths' Peer Relationships: Experiences, Expectations, and Implications for WelluBeing. Journal of Research on Adolescence, 14(3), 313-340. 
Earle, L. \& Earle, T. (1999). Social Context Theory. South Pacific Journal of Psychology, 11(2), 1-12. http://spjp.massey.ac.nz/ issues/1999-v11/v11-2_earle.pdf (Accessed 12/02/2017)

Elia, J. (1994). Homophobia in the High School: A Problem in Need of a Resolution. The High School Journal, 77(1/2), 177-185.

Ensel, W., \& Lin, N. (1991). The Life Stress Paradigm and Psychological Distress. Journal of Health and Social Behavior, 32(4), 321-341.

Fenzel, L. M. (1989a). Role strain in early adolescence: A model for investigating school transition stress. Journal of Early Adolescence, 9, 13-33.

Fenzel, L. M. (1989b). Role strains and the transition to middle school: Longitudinal trends and sex differences. Journal of Early Adolescence, 9, 211-226.

Fenzel, L. M. (2000). Prospective study of changes in global selfworth and strain during the transition to middle school. Journal of Early Adolescence, 20, 93-116.

Fetzer Institute. (1999). Multidimensional measurement of religiousness/spirituality for use in healthcare research: A report of the Fetzer Institute/National Institute on Aging Workgroup. Kalamazoo, MI: John E. Fetzer Institute.

Floyd, F., Stein, J., Harter, T., Allison, S., \& Nye, K. (1999). Gay, Lesbian, and Bisexual Youths: Separation-Individuation, Parental Attitudes, Identity Consolidation, and Well-Being. Journal of Youth and Adolescence, 28(6), 719-739.

Frost, D. M., \& Meyer, I. H. (2009). Internalized Homophobia and Relationship Quality among Lesbians, Gay Men, and Bisexuals. Journal of Counseling Psychology, 56(1), 97-109.

Furman, W. (1996). The measurement of children and adolescents' perceptions of friendships: Conceptual and methodological issues. In W. M. Bukowski, A. F. Newcomb, \& W. W. Hartup (Eds.), The company they keep: Friendships in childhood and adolescence (pp. 41-65). Cambridge, UK: Cambridge University Press.

Garnets, L., Herek, G. M., \& Levy, B. (1990). Violence and Victimization of Lesbians and Gay Men: Mental Health Consequences. Journal of Interpersonal Violence, 5, 366-383.

Garofalo, R., Wolf, R. C., Kessel, S., Palfrey, J., \& DuRant, R. H. (1998). The association between health risk behaviors and sexual orientation among a school-based sample of adolescents. Pediatrics, 101(5), 895-902.
Goldberg, D. (1972). The detection of psychiatric illness by questionnaire. London: Oxford University Press.

Grossman, A. (1997). Growing Up with a "Spoiled Identity". Journal of Gay \& Lesbian Social Services, 6(3), 45-56.

*Grossman, A., \& Kerner, M. (1998). Self-Esteem and Supportiveness as Predictors of Emotional Distress in Gay Male and Lesbian Youth. Journal of Homosexuality, 35(2), 25-39.

Harter, S. (1982). The perceived competence scale for children. Child Development, 53, 87-97.

Herek, G. M., Gillis, J. R., \& Cogan, J. C. (2009). Internalized Stigma among Sexual Minority Adults: Insights from a Social Psychological Perspective. Journal of Counseling Psychology, 56(1), 32-43.

*Hershberger, S., \& D’Augelli, A. (1995). The Impact of Victimization on the Mental Health and Suicidality of Lesbian, Gay, and Bisexual Youths. Developmental Psychology, 31(1), 65-74.

Hetrick, E., \& Martin, A. (1987). Developmental Issues and Their Resolution for Gay and Lesbian Adolescents. Journal of Homosexuality, 14(1), 25-43.

Higgins, E., \& Hoffman, Martin L. (1987). Self-Discrepancy: A Theory Relating Self and Affect. Psychological Review, 94(3), 319340.

*loverno, S., Belser, A. B., Baiocco, R., Grossman, A. H., Russell, S. T. (2016). The Protective Role of Gay-Straight Alliances for Lesbian, Gay, Bisexual, and Questioning Students: A Prospective Analysis. Psychology of Sexual Orientation and Gender Diversity, 3(4), 397-406.

Jackson, S. D., \& Mohr, J. J. (2016). Conceptualizing the Closet: Differentiating Stigma Concealment and Nondisclosure Processes. Psychology of Sexual Orientation and Gender Diversity, 3(1), 80-92.

Johnson, J. H., \& McCutcheon, S. M. (1980). Assessing life stress in older children and adolescents: Preliminary findings with the Life Events Checklist. In I. G. Sarason \& C. D. Spielberger (Eds.), Stress and anxiety (Vol. 6, pp. 111-125). New York: Wiley.

Jordan, K. M., Vaughan, J. S., \& Woodworth, K. J. (1997). I Will Survive. Journal of Gay \& Lesbian Social Services, 7(4), 17-33.

Kinsey, A. C., Pomeroy, W. B., \& Martin, C. E. (1948). Sexual Behavior in the Human Male. Philadelphia: W.B. Saunders. 
Kosciw, J. G., Greytak, E. A., Bartkiewicz, M. J., Boesen, M. J., \& Palmer, N. A. (2012). The 2011 National School Climate Survey: The experiences of lesbian, gay, bisexual and transgender youth in our nation's schools. New York, NY: Gay, Lesbian and Straight Education Network. http://www.glsen.org/sites/default/ files $/ 2011 \% 20$ National $\% 20$ School $\% 20$ Climate $\% 20$ Survey $\% 20$ Full\%20Report.pdf (Accessed 12/07/2017)

Lee, R. M., \& Robbins, S. B. (1995). Measuring belongingness: The social connectedness and the social assurance scales. Journal of Counseling Psychology, 42, 232-241.

Lee, R. M., \& Robbins, S. B. (1998). The Relationship Between Social Connectedness and Anxiety, Self-Esteem, and Social Identity. Journal of Counseling Psychology, 45(3), 338-345.

Legate, N., Ryan, R., \& Weinstein, N. (2012). Is Coming Out Always a "Good Thing"? Exploring the Relations of Autonomy Support, Outness, and Wellness for Lesbian, Gay, and Bisexual Individuals. Social Psychological and Personality Science, 3(2), 145-152.

Lewis, R. J., Derlega, V. J., Berndt, A., Morris, L.M., \& Rose, S. (2001). An empirical

analysis of stressors for gays and lesbians. Journal of Homosexuality, 42, 63-89.

Luhtanen, R., \& Crocker, J. (1992). A collective self-esteem scale: Self-evaluation of one's social identity. Personality and Social Psychology Bulletin, 18, 302-318.

Mapou, R. L., Ayres, J., \& Cole, S. P. (1983). An analysis of problem areas and counseling experiences of gay white males. American Journal of Community Psychology, 11, 323-336.

Marsh, H. W., \& O'Neill, R. (1984). Self-Description Questionnaire III: The Construct Validity of Multidimensional Self-Concept Ratings by Late Adolescents. Journal of Educational Measurement, 21(2), 153-74.

Marsiglio, W. (1993). Attitudes toward Homosexual Activity and Gays as Friends: A National Survey of Heterosexual 15- to 19-YearOld Males. The Journal of Sex Research, 30(1), 12-17.

Martin-Storey, A. (2015). Prevalence of Dating Violence Among Sexual Minority Youth: Variation Across Gender, Sexual Minority Identity and Gender of Sexual Partners. Journal of Youth and Adolescence, 44(1), 211-224.

McNicholas, S. L. (2002). Social support and positive health practices. Western Journal of Nursing Research, 24(7), 772-87.
Meyer, I. (1995). Minority Stress and Mental Health in Gay Men. Journal of Health and Social Behavior, 36(1), 38-56.

Moher, D., Liberati, A., Tetzlaff, J., Altman, D. G., The PRISMA Group (2009). Preferred Reporting Items for Systematic Reviews and Meta-Analyses: The PRISMA Statement. PLoS Med 6(7): e1000097. doi:10.1371/journal.pmed1000097

Mohr, J., \& Fassinger, R. (2000). Measuring Dimensions of Lesbian and Gay Male Experience. Measurement and Evaluation in Counseling and Development, 33(2), 66-90.

Murdoch University (2019), Systematic Reviews: Using PICO or PICo [online]. Available at: https://libguides.murdoch.edu.au/ systematic/PICO [Accessed 22 Feb. 2019].

Nungesser, L. (1983). Homosexual acts, actors, and identities. New York: Praeger.

O'Conor, A. (1994). Who Gets Called Queer in School? Lesbian, Gay and Bisexual Teenagers, Homophobia and High School. The High School Journal, 77(1/2), 7-12.

O'Moore, M., \& Kirkham, C. (2001). Self-Esteem and Its Relationship to Bullying Behaviour. Aggressive Behavior, 27(4), 269-283.

Overbeek, G., Zeevalkink, H., Vermulst, A., \& Scholte, R. H. J. (2010). Peer Victimization, Self-Esteem, and Ego Resilience Types in Adolescents: A Prospective Analysis of Person-Context Interactions. Social Development, 19(2), 270-284.

Pachankis, J. E. (2007). The Psychological Implications of Concealing a Stigma: A Cognitive-Affective-Behavioral Model. Psychological Bulletin, 133(2), 328-345.

Pachankis, J. E., Cochran, S. D., \& Mays, V. M. (2015). The Mental Health of Sexual Minority Adults In and Out of the Closet: A Population-Based Study. Journal of Consulting and Clinical Psychology, 83(5), 890-901.

Peplau, L. A., \& Fingerhut, A. W. (2007). The Close Relationships of Lesbians and Gay Men. 58, 405-424.

Pilkington, N. W., \& D'Augelli, A. R. (1995). Victimization of lesbian, gay, and bisexual youth in community settings. Journal of Community Psychology, 23, 34-56.

*Poteat, V. P., Yoshikawa, H., Calzo, J. P., Gray, M. L., DiGiovanni, C. D., Lipkin, A., Mundy-Shephard, A., Perrotti, J., Scheer, J. R., Shaw, M. P. (2015). Contextualizing Gay $\square$ Straight Alliances: 
Student, Advisor, and Structural Factors Related to Positive Youth Development Among Members. Child Development, 86(1), 176193.

Procidano, M. E., \& Heller, K. (1983). Measures of perceived social support from friends and from family: Three validation studies. American Journal of Community Psychology, 11, 1-24.

Radloff, L. S. (1977). The CES-D scale: A self-report depression scale for research in the general population. Journal of Applied Psychological Measures, 3, 385-401.

Remafedi, G. (1987). Adolescent homosexuality psychosocial and medical implications. Pediatrics, 79(3), 331-337.

Riggle, E., Rostosky, S., Black, W., \& Rosenkrantz, D. (2017). Outness, Concealment, and Authenticity: Associations With LGB Individuals' Psychological Distress and Well-Being. Psychology of Sexual Orientation and Gender Diversity, Psychology of Sexual Orientation and Gender Diversity, 2016.

Rosario, M., Schrimshaw, E. W., Hunter, J., \& Gwadz, M. (2002). Gay-related stress and emotional distress among gay, lesbian, and bisexual youths: A longitudinal examination. Journal of Consulting and Clinical Psychology, 70, 967-975.

*Rosario, M., Schrimshaw, E. W., \& Hunter, J. (2005). Psychological Distress Following Suicidality among Gay, Lesbian, and Bisexual Youths: Role of Social Relationships. Journal of Youth and Adolescence, 34(2), 149-161.

*Rosario, M., Schrimshaw, E. W., \& Hunter, J. (2011). Different Patterns of Sexual Identity Development over Time: Implications for the Psychological Adjustment of Lesbian, Gay, and Bisexual Youths. Journal of Sex Research, 48(1), 3-15.

Rosenberg, M. (1965). Society and the adolescent self-image. Princeton, NJ: Princeton University Press.

Rosenberg, M. (1979). Conceiving the self. New York, NY: Basic Books.

Rosenberg, M. (1989). Self-concept research: A historical overview. Social Forces, 68, 34-44.

Rosenberg, M., Schooler, C., Schoenbach, C., \& Rosenberg, F. (1995). Global self-esteem and specific self-esteem: Different concepts, different outcomes. American Sociological Review, 60 , 141-156. doi:10.2307/2096350
Rosenfield, S., \& Wenzel, S. (1997). Social networks and chronic mental illness: A test of four perspectives. Social Problems, 44(2), 200.

*Rotheram-Borus, M., Rosario, M., Van Rossem, R., Reid, H., Gillis, R., Zahn-Waxler, Carolyn, \& Patterson, Charlotte J. (1995). Prevalence, Course, and Predictors of Multiple Problem Behaviors Among Gay and Bisexual Male Adolescents. Developmental Psychology, 31(1), 75-85.

Russell, S. T., \& Joyner, K. (2001). Adolescent sexual orientation and suicide risk: Evidence from a national study. American Journal of Public Health, 91(8), 1276-81.

Russell, S. T., Ryan, C., Toomey, R. B., Diaz, R. M., \& Sanchez, J. (2011). Lesbian, Gay, Bisexual, and Transgender Adolescent School Victimization: Implications for Young Adult Health and Adjustment. Journal of School Health, 81(5), 223-230.

*Russell, S. T., Toomey, R. B., Ryan, C., \& Diaz, R. M. (2014). Being Out at School: The Implications for School Victimization and Young Adult Adjustment. American Journal of Orthopsychiatry, 84(6), 635-643.

Ryan, C., Huebner, D., Diaz, R. M., \& Sanchez, J. (2009). Family rejection as a predictor of negative health outcomes in white and Latino lesbian, gay, and bisexual young adults. Pediatrics, 123, 346-352. doi:10.1542/peds.2007-3524

Ryan, C., Russell, S. T., Huebner, D., Diaz, R., \& Sanchez, J. (2010). Family acceptance in adolescence and the health of LGBT young adults. Journal of Child and Adolescent Psychiatric Nursing, 23, 205-213. doi:10.1111/j.1744-6171.2010. 00246.x

Ryff, C. D. (1989). Happiness is everything, or is it? Explorations on the meaning of psychological well-being. Journal of Personality and Social Psychology, 57, 1069-1081.

Sarason, B. R., Shearin, E. N., Pierce, G. R., \& Sarason, I. G. (1987). Interrelations of Social Support Measures: Theoretical and Practical Implications. Journal of Personality and Social Psychology, 52(4), 813-832.

*Savin-Williams, R. (1989). Coming Out to Parents and SelfEsteem Among Gay and Lesbian Youths. Journal of Homosexuality, 18(1-2), 1-35.

Savin-Williams, R. C. (1990). Gay and lesbian youth: Expressions of identity. New York: Hemisphere. 
Savin-Williams, R., Beutler, Larry E., \& Rothblum, Esther D. (1994). Verbal and Physical Abuse as Stressors in the Lives of Lesbian, Gay Male, and Bisexual Youths: Associations With School Problems, Running Away, Substance Abuse, Prostitution, and Suicide. Journal of Consulting and Clinical Psychology, 62(2), 261269.

Savin-Williams, R., \& Diamond, C. (2000). Sexual Identity Trajectories Among Sexual-Minority Youths: Gender Comparisons. Archives of Sexual Behavior, 29(6), 607-627.

Schuck, K. D., \& Liddle, B. J. (2001). Religious Conflicts Experienced by Lesbian, Gay, and Bisexual Individuals. Journal of Gay \& Lesbian Psychotherapy, 5(2), 63-82.

Sherkat, D. E. (2002). Sexuality and Religious Commitment in the United States: An Empirical Examination. Journal for the Scientific Study of Religion, 41(2), 313-323.

Shidlo, A. (1994). Internalized homophobia: Conceptual and empirical issues in measurement. In B. Greene \& G. M. Herek (Eds.), Lesbian and gay psychology: Theory, research, and clinical applications (pp. 176-205). Thousand Oaks, CA: Sage.

Silverschanz, P., Cortina, L. M., Kornik, J., \& Magley, V. J. (2008). Slurs, snubs, and queer jokes: Incidence and impact of heterosexist harassment in academia. Sex Roles, 58, 179-191. doi: 10.1007/s11199-007-9329-7

*Snapp, S., Watson, R., Russell, S., Diaz, R., \& Ryan, C. (2015). Social Support Networks for LGBT Young Adults: Low Cost Strategies for Positive Adjustment. Family Relations, 64(3), 420430.

Sophie, J. (1986). A critical examination of stage theories of lesbian identity development. Journal of Homosexuality, 12, 39-51.

Spitzer, R. L., Kroenke, K., Williams, J. B., \& L“owe, B. (2006). A brief measure for assessing generalized anxiety disorder: The GAD-7. Archives of Internal Medicine, 166, 1092-1097. doi:10.1001/ archinte.166.10.1092

Stattin, H., \& Kerr, M. (2000). Parental monitoring: A reinterpretation. Child Development, 71, 1072-1085.

Szymanski, D. M., \& Gupta, A. (2009). Examining the Relationship between Multiple Internalized Oppressions and African American Lesbian, Gay, Bisexual, and Questioning Persons' Self-Esteem and Psychological Distress. Journal of Counseling Psychology, 56(1), 110-118.
Thoits, P. (1985). Self-Labeling Processes in Mental Illness: The Role of Emotional Deviance. American Journal of Sociology, 91(2), 221-249.

*Toomey, R., Ryan, C., Diaz, R., \& Russell, S. (2011). High School Gay-Straight Alliances (GSAs) and Young Adult Well-Being: An Examination of GSA Presence, Participation, and Perceived Effectiveness. Applied Developmental Science, 15(4), 175-185.

Van Heeringen, C., \& Vincke, J. (2000). Suicidal acts and ideation in homosexual and bisexual young people: A study of prevalence and risk factors. Social Psychiatry and Psychiatric Epidemiology, 35(11), 494-499.

Vincke, J., \& Bolton, R. (1996). The social support of gay men: an explorative study. Journal of Homosexuality, 31(4), 107-121.

*Vincke, J., Van Heeringen, K., Coyle, Adrian, \& Wilkinson, Sue. (2002). Confidant support and the mental wellbeing of lesbian and gay young adults: A longitudinal analysis. Journal of Community \& Applied Social Psychology, 12(3), 181-193.

Voelkl, K. E. (1996a). Identification with school. American Journal of Education, 105, 294-318.

Voelkl, K. E. (1996b). Measuring students' identification with school. Educational and Psychological Measurement, 56, 760-770.

Von Elm, E., Altman, D. G., Egger, M., Pocock, S. J., Gøtzsche, P. C., Vandenbroucke, J. P., \& Strobe Initiative. (2007). The Strengthening the Reporting of Observational Studies in Epidemiology (STROBE) statement: guidelines for reporting observational studies. Preventive medicine, 45(4), 247-251.

Waldo, C. R., Hesson-McInnis, M. S., \& D'Augelli, A. R. (1998). Antecedents and Consequences of Victimization of Lesbian, Gay, and Bisexual Young People: A Structural Model Comparing Rural University and Urban Samples. American Journal of Community Psychology, 26(2), 307-334.

*Wilkerson, J. M., Schick, V. R., Romijnders, K. A., Bauldry, J., \& Butame, S. A. (2017). Social Support, Depression, Self-Esteem, and Coping Among LGBTQ Adolescents Participating in Hatch Youth. Health Promotion Practice, 18(3), 358-365.

Woodford, M. R., Han, Y., Craig, S., Lim, C., \& Matney, M. M. (2014). Discrimination and mental health among sexual minority college students: The type and form of discrimination does matter. Journal of Gay \& Lesbian Mental Health, 18(2), 142-163. doi: $10.1080 / 19359705.2013 .833882$ 
Woodford, M. R., Howell, M. L., Silverschanz, P., \& Yu, L. (2012). "That's so gay!": Examining the covariates of hearing this expression among gay, lesbian, and bisexual college students. Journal of American College Health, 60(6), 429-434. doi: 10.1080/07448481.2012.673519

*Woodford, M. R., Paceley, M. S., Kulick, A., \& Hong, J. S. (2015). The LGBQ Social Climate Matters: Policies, Protests, and Placards and Psychological Well-Being Among LGBQ Emerging Adults. Journal of Gay \& Lesbian Social Services, 27(1), 116-141.

Zimet, G. D., Dahlem, N. W., Zimet, S. G., \& Farley, G. K. (1988). The Multidimensional Scale of Perceived Social Support. Journal of Personality Assessment, 52, 30-41. 